\title{
The Howe dual pair in Hermitean Clifford analysis
}

\author{
Fred Brackx, Hennie De Schepper, David Eelbode \\ and Vladimir Souček
}

\begin{abstract}
Clifford analysis offers a higher dimensional function theory studying the null solutions of the rotation invariant, vector valued, first order Dirac operator $\partial$. In the more recent branch Hermitean Clifford analysis, this rotational invariance has been broken by introducing a complex structure $J$ on Euclidean space and a corresponding second Dirac operator $\partial_{J}$, leading to the system of equations $\partial f=0=\partial_{J} f$ expressing so-called Hermitean monogenicity. The invariance of this system is reduced to the unitary group. In this paper we show that this choice of equations is fully justified. Indeed, constructing the Howe dual for the action of the unitary group on the space of all spinor valued polynomials, the generators of the resulting Lie superalgebra reveal the natural set of equations to be considered in this context, which exactly coincide with the chosen ones.
\end{abstract}

\section{Introduction}

The aim of the paper is to analyse the effect and consequences of adding to the standard setting of Clifford analysis a new datum, a so-called complex structure, in this way establishing a closer connection with complex analysis and Kähler geometry, as opposed to the Riemannian setting for classical Clifford analysis.

Let us consider a Euclidean space $E$ of dimension $m \geq 3$, carrying a positive definite scalar product $B(.,$.$) . Concepts and definitions are first in-$ troduced in a co-ordinate free way; the computations needed may then be executed in any orthonormal frame, making it clear that all results obtained

2000 Mathematics Subject Classification: 22E46, 30G35, 15 A66.

Keywords: Hermitean Clifford analysis, Howe dual pair. 
are independent of the choice of the orthonormal basis. Functions in Clifford analysis are defined in $E$ and have their values either in the corresponding Clifford algebra, or in a spinor representation, see [3, 10, 14, 17]. Here we consider the case of spinor valued functions. As usual, let $\mathrm{SO}(E)$ be the group of orientation preserving automorphisms of $E$ leaving the scalar product invariant. The group $\mathrm{SO}(E)$ is doubly covered by $\operatorname{Spin}(E)$, a subgroup of the Clifford group, which may be identified with the double cover $\operatorname{Spin}(m)$ of $\mathrm{SO}(m)$, when choosing an orthonormal basis $\left\{e_{j}\right\}_{j=1}^{m}$ in $E$. Clifford analysis, in its standard Euclidean setting, studies monogenic functions, i.e. the null solutions of the $\mathrm{SO}(E)$-invariant Dirac operator $\partial$. In co-ordinates with respect to the chosen basis $\left\{e_{j}\right\}_{j=1}^{m}$ this Dirac operator is given by $\partial=\sum_{j} e_{j} \partial_{j}$.

In the books $[21,9]$ and the series of papers $[22,7,11,1,2]$ so-called Hermitean Clifford analysis emerged as a refinement of Euclidean Clifford analysis. Hermitean Clifford analysis is based on the introduction of an additional datum, a so-called complex structure, in order to bring the notion of monogenicity closer to complex analysis. Its function theory is still in full development, see $[6,23,8,4,5]$. A complex structure $J$ on $E$ should be compatible with the Euclidean structure on $E$, i.e. $J \in \mathrm{SO}(E)$, ánd $J^{2}=-\mathbf{1}_{E}$, whence it is seen at once that the dimension of $E$ is forced to be even: $m=2 n$. The subgroup of $\mathrm{SO}(E)$ preserving the complex structure -i.e. commuting with $J$ - turns out to be isomorphic with $\mathrm{U}(n)$ (see [1]). The complex structure $J$ induces an associated Dirac operator $\partial_{J}$. Hermitean Clifford analysis then focusses on Hermitean monogenic functions, i.e. simultaneous null solutions of both operators $\partial$ and $\partial_{J}$, in this way breaking down the rotational invariance of the Dirac operator, reducing it to $\mathrm{U}(n)$-invariance for the considered system.

The central topic dealt with in this paper concerns a justification for these equations, based on the study of the space of spinor valued polynomials on $\mathbb{R}^{2 n}$. Under the action of $\mathrm{U}(n)$, this space decomposes into a sum of irreducible subspaces, which is however not multiplicity free: in fact, the irreducible pieces all appear with infinite multiplicity. The idea behind the Howe dual pair is to complement the $\mathrm{U}(n)$-action by a new, hidden, symmetry commuting with it, in such a way that the resulting decomposition becomes multiplicity free. Such problems are well-known in representation theory for the spaces of scalar valued polynomials under the respective actions of the groups $\mathrm{SO}(m)$ and $\mathrm{U}(n)$ (see the review paper [16]), or for polynomials with values in a Grassman algebra (see [19]). To our knowledge there is no similar treatment available for the case of spinor valued polynomials, although in [20, p. 205] the case of physical dimension four is mentioned. The main aim of the paper is to analyze the Howe dual pair relevant for Hermitean Clifford analysis, i.e. the case of spinor-valued poly- 
nomials with the action of the symmetry group preserving a chosen complex structure, being a special case of an abstract formulation of Howe dual pairs in [20]. We describe the corresponding Howe dual pair in detail (see Theorem 4), including an explicit parametrization of representations appearing in the theorem on separation of variables. We show that the decompositions obtained exactly correspond to the so-called Fischer decompositions for Hermitean monogenic functions (see [12]). As a by-product we determine the natural space of functions to be considered in the present setting, which eventually turns out to coincide exactly with the kernel of the pair of differential operators studied in Hermitean Clifford analysis.

For the reader's convenience, we start with the formulation of the Howe dual pair and the theorem of separation of variables for two simpler, classical cases of scalar-valued polynomials (Theorems 1 and 2), the corresponding proofs showing the appropriate scheme to be followed, also for the proofs of more complicated cases. Next we pass to the case of standard Euclidean Clifford analysis, where the Fischer decomposition for spinor-valued functions is at the very heart of this function theory. We explicitly describe the appropriate Howe dual pair for this case and we show its relation to the Fischer decomposition (Theorem 3). Finally, we arrive at the desired case of Hermitean Clifford analysis. Observe that, in the proof of Theorem 4, we could have used abstract results from [19] and restricted ourselves to the computation of the missing explicit parametrization for this case. It is, however, much simpler (and more convenient from the present point of view) to present a direct proof of the theorem without referring to the abstract scheme of [19]. To make the paper self-contained a short section is devoted to the basics of Clifford algebra.

\section{Clifford algebra: the basics}

Consider a real vector space $E$ of dimension $m$, equipped with a symmetric, positive definite, real-bilinear form $B(X, Y), X, Y \in E$ with associated quadratic form $Q(X)=B(X, X)$. The orthogonal and special orthogonal groups $\mathrm{O}(E)$ and $\mathrm{SO}(E)$ are defined as usual, as the groups of automorphisms, respectively orientation preserving automorphisms, $g \in \operatorname{Aut}(E)$ leaving the bilinear form $B$ invariant:

$$
B(g X, g Y)=B(X, Y), \forall X, Y \in E
$$

Now, let $\left(e_{1}, \ldots, e_{m}\right)$ be a basis of $E$ which we assume to be orthonormal w.r.t. the bilinear form $B$, i.e. $B\left(e_{j}, e_{k}\right)=\delta_{j k}, j, k=1, \ldots, m$. The introduction of this basis leads to the identification $\mathrm{O}(E) \simeq \mathrm{O}(m)$, through 
representation by $(m \times m)$-matrices $g=\left[g_{j k}\right]$, naturally satisfying the condition $g g^{T}=g^{T} g=\mathbf{1}_{m}$ with $\mathbf{1}_{m}$ the unit matrix of order $m$, while in the case of $\mathrm{SO}(E) \simeq \mathrm{SO}(m)$, the additional condition $\operatorname{det}(g)=1$ is imposed as well.

Turning to the complexification $E_{\mathbb{C}}$ of the vector space $E$, as well as the complexification $B_{\mathbb{C}}$ of the bilinear form $B$, let us now consider the Clifford algebras $\mathcal{C} \ell(E,-Q)$ over $E$ and $\mathcal{C} \ell\left(E_{\mathbb{C}},-Q_{\mathbb{C}}\right)$ over $E_{\mathbb{C}}$, where the Clifford or geometric product is associative but non-commutative. With respect to the chosen basis, it is governed the rules

$$
e_{j}^{2}=-1, j=1, \ldots, m, \quad e_{j} e_{k}+e_{k} e_{j}=0, j \neq k=1, \ldots, m
$$

In standard Euclidean Clifford analysis, we associate with each vector $X \in E$ with components $\left(X_{1}, \ldots, X_{m}\right) \in \mathbb{R}^{m}$ the real Clifford vector $\underline{X}=\sum_{j=1}^{m} X_{j} e_{j}$. Its Fischer dual is the first order Clifford vector valued differential operator $\partial=\sum_{j=1}^{m} e_{j} \partial_{X_{j}}$, called the Dirac operator, which may also be obtained in a co-ordinate free way as a generalized gradient, see e.g. [1, 2]. It is precisely this Dirac operator which underlies the notion of monogenicity, a notion which is the higher dimensional counterpart of holomorphy in the complex plane. A smooth function $f$, defined on $E$ or on $E_{\mathbb{C}}$ and taking values in either the real Clifford algebra $\mathcal{C} \ell(E,-Q)$ or the complex Clifford algebra $\mathcal{C} \ell\left(E_{\mathbb{C}},-Q_{\mathbb{C}}\right)$, is called left monogenic if it fulfills the Dirac equation $\partial[f]=0$.

It is well-known that the groups $\mathrm{O}(E)$ and $\mathrm{SO}(E)$ are doubly covered by the so-called pin group $\operatorname{Pin}(E)$ and spin group $\operatorname{Spin}(E)$ of the Clifford algebra respectively, given by

$$
\operatorname{Pin}(E)=\left\{s \in \mathcal{C} \ell(E,-Q): \exists k \in \mathbb{N}, s=\underline{\omega}_{1} \ldots \underline{\omega}_{k}, \underline{\omega}_{i} \in S^{m-1}, i=1 \ldots k\right\}
$$

and

$\operatorname{Spin}(E)=\left\{s \in \mathcal{C} \ell(E,-Q): \exists k \in \mathbb{N}, s=\underline{\omega}_{1} \ldots \underline{\omega}_{2 k}, \underline{\omega}_{i} \in S^{m-1}, i=1 \ldots 2 k\right\}$

where $S^{m-1}$ is the unit sphere in $E$; through co-ordinatization it holds that $\operatorname{Pin}(E) \simeq \operatorname{Pin}(m)$ and $\operatorname{Spin}(E) \simeq \operatorname{Spin}(m)$. Taking $g \in \operatorname{SO}(E)$, with corresponding spin element $s_{g} \in \operatorname{Spin}(E) \simeq \operatorname{Spin}(m)$, the action of $g$ on vectors in $E$ is expressed in Clifford language as

$$
X^{\prime}=g[X] \longleftrightarrow \underline{X}^{\prime}=s_{g} \underline{X} s_{g}^{-1}
$$

It follows that the Dirac operator is invariant under the special orthogonal group action, or, equally, under the action of $\operatorname{Spin}(m)$, which in Clifford language has the following explicit form: if $s \in \operatorname{Spin}(m)$ and $H(s)$ is its socalled $H$-representation, given for a Clifford algebra valued function $F$ by

$$
H(s)[F(\underline{X})]=s F\left(s^{-1} \underline{X} s\right) s^{-1}
$$

then one has the commutation relation $[\partial, H(s)]=0$. A similar observation applies to $\operatorname{Pin}(E)$. 
We now introduce the building blocks of the Hermitean Clifford setting. We endow the space $(E, B)$ with a so-called complex structure by choosing an $\mathrm{SO}(E)$ element $J$ for which $J^{2}=-\mathbf{1}$ and creating in this way the Hermitean space $(E, B, J)$. Clearly $(\operatorname{det} J)^{2}=(-1)^{m}$, forcing the dimension $m$ of $E$ to be even: in the present context we thus put $m=2 n$.

In the complexified space $\left(E_{\mathbb{C}}, B_{\mathbb{C}}\right)$ the projection operators $\frac{1}{2}(\mathbf{1} \pm i J)$ are considered, creating two subspaces of $E_{\mathbb{C}}$, viz

$$
W^{ \pm}=\left\{Z^{ \pm} \in E_{\mathbb{C}}: Z^{ \pm}=\frac{1}{2}(\mathbf{1} \pm i J) X, X \in E\right\}
$$

which are isotropic with respect to the bilinear form $B_{\mathbb{C}}$ and constitute the direct sum decomposition $E_{\mathbb{C}}=W^{+} \oplus W^{-}$. Extending the action of $g \in \mathrm{SO}(E)$ to vectors in $E_{\mathbb{C}}$ by $Z^{ \pm} \in W^{ \pm} \mapsto g\left[Z^{ \pm}\right]=\frac{1}{2}(g[X] \pm i g[J X])$, the isotropic subspaces $W^{ \pm}$of $E_{\mathbb{C}}$ are seen to remain invariant if and only if $g$ commutes with the complex structure $J$, or in other words, if $g$ belongs to

$$
\mathrm{SO}_{J}(E)=\{g \in \mathrm{SO}(E): g J=J g\}
$$

Similarly, one defines $\mathrm{O}_{J}(E) \subset \mathrm{O}(E)$. Defining a Hermitean inner product on $E_{\mathbb{C}}$ by

$$
(Z, U)=B_{\mathbb{C}}\left(\left(\frac{1}{2}(\mathbf{1} \pm i J) X\right)^{c}, \frac{1}{2}(\mathbf{1} \pm i J) Y\right)
$$

for $Z=\frac{1}{2}(\mathbf{1} \pm i J) X, U=\frac{1}{2}(\mathbf{1} \pm i J) Y, X, Y \in E$, we moreover have that this inner product is preserved by the group action of $\mathrm{SO}_{J}(E)$ (as well as by the one of $\left.\mathrm{O}_{J}(E)\right)$.

Observe that the orthonormal basis $\left(e_{1}, \ldots, e_{2 n}\right)$ of $E$ may always be chosen in such a way that the complex structure $J \in \mathrm{SO}(E)$ is represented by the matrix

$$
J=\left[\begin{array}{cc}
0 & \mathbf{1}_{n} \\
-\mathbf{1}_{n} & 0
\end{array}\right]
$$

For an arbitrary element in $\mathrm{SO}_{J}(E)$, the commutation relation with $J$ is then reflected in the specific form of the corresponding matrix as follows:

$$
A=\left[\begin{array}{rr}
B & C \\
-C & B
\end{array}\right]
$$

with $B B^{T}+C C^{T}=\mathbb{E}$ and $B C^{T}-C B^{T}=0$. These conditions on the submatrices $B$ and $C$ imply that $B \pm i C$ both belong to the unitary group $\mathrm{U}(n)$. In other words: the subgroup

$$
\mathrm{SO}_{J}(2 n)=\{A \in \mathrm{SO}(2 n): A J=J A\}
$$

is isomorphic with $\mathrm{U}(n)$, and so is $\mathrm{SO}_{J}(E)$. 
Note that, by means of the projection operators $\frac{1}{2}(\mathbf{1} \pm i J)$, the basis $\left(e_{1}, \ldots, e_{2 n}\right)$ also gives rise to an alternative basis for $E_{\mathbb{C}}$ :

$$
\begin{aligned}
& \mathfrak{f}_{j}=\frac{1}{2}(\mathbf{1}+i J)\left[e_{j}\right]=\frac{1}{2}\left(e_{j}-i e_{n+j}\right), j=1, \ldots, n \\
& \mathfrak{f}_{j}^{\dagger}=-\frac{1}{2}(\mathbf{1}-i J)\left[e_{j}\right]=-\frac{1}{2}\left(e_{j}+i e_{n+j}\right), \quad j=1, \ldots, n
\end{aligned}
$$

called the Witt basis; it naturally splits into separate bases $\left(\mathfrak{f}_{1}, \ldots, \mathfrak{f}_{n}\right)$ and $\left(\mathfrak{f}_{1}^{\dagger}, \ldots, \mathfrak{f}_{n}^{\dagger}\right)$ for $W^{+}$and $W^{-}$, respectively. The Witt basis elements satisfy the Grassmann relations

$$
\mathfrak{f}_{j} \mathfrak{f}_{k}+\mathfrak{f}_{k} \mathfrak{f}_{j}=0, \quad \mathfrak{f}_{j}^{\dagger} \mathfrak{f}_{k}^{\dagger}+\mathfrak{f}_{k}^{\dagger} \mathfrak{f}_{j}^{\dagger}=0, \quad j, k=1, \ldots, n
$$

from which also their isotropy follows, and the duality relations

$$
\mathfrak{f}_{j} \mathfrak{f}_{k}^{\dagger}+\mathfrak{f}_{k}^{\dagger} \mathfrak{f}_{j}=\delta_{j k}, \quad j, k=1, \ldots, n
$$

The $\nmid$-notation above corresponds to a Hermitean conjugation in $\mathcal{C} \ell\left(E_{\mathbb{C}}\right.$, $\left.-Q_{\mathbb{C}}\right)$, defined as follows. Take $\mu \in \mathcal{C} \ell\left(E_{\mathbb{C}},-Q_{\mathbb{C}}\right)$ arbitrarily, with $\mu=a+i b$, where $a, b \in \mathcal{C} \ell(E,-Q)$. Then $\mu^{\dagger}=\bar{a}-i \bar{b}$ where $\bar{a}$ and $\bar{b}$ denote the traditional Clifford conjugates of $a$ and $b$ in $\mathcal{C} \ell(E,-Q)$.

The components of the vector $X$ are now denoted as $\left(x_{1}, \ldots, x_{n}, y_{1}, \ldots, y_{n}\right)$, and the corresponding Clifford vector $\underline{X}$ may thus be rewritten in terms of the Witt basis as

$$
\underline{X}=\sum_{j=1}^{n}\left(x_{j} e_{j}+y_{j} e_{n+j}\right)=\sum_{j=1}^{n}\left(z_{j} \mathfrak{f}_{j}-z_{j}^{c} \mathfrak{f}_{j}^{\dagger}\right)
$$

where we have introduced the complex variables $z_{j}=x_{j}+i y_{j}$ and their complex conjugates $z_{j}^{c}, j=1, \ldots, n$. For vectors in the isotropic subspaces $W^{ \pm}$of $E_{\mathbb{C}}$ a similar identification results into

$$
\begin{aligned}
& Z^{+}=\frac{1}{2}(\mathbf{1}+i J) X \quad \longleftrightarrow \quad \underline{z}=\sum_{j=1}^{n} z_{j} \mathfrak{f}_{j} \\
& Z^{-}=\frac{1}{2}(\mathbf{1}-i J) X \quad \longleftrightarrow-\underline{z}^{\dagger}=-\sum_{j=1}^{n} z_{j}^{c} \mathfrak{f}_{j}^{\dagger}
\end{aligned}
$$

such that the relation $X=Z^{+}+Z^{-}$may be rewritten in Clifford language as $\underline{X}=\underline{z}-\underline{z}^{\dagger}$.

In this way, we have also arrived at the definition of the Hermitean Dirac operators

$$
\partial_{\underline{z}}=\sum_{j=1}^{n} \mathfrak{f}_{j}^{\dagger} \partial_{z_{j}} \quad \text { and } \quad \partial_{\underline{z}^{\dagger}}=\sum_{j=1}^{n} \mathfrak{f}_{j} \partial_{z_{j}^{c}}=\partial_{\underline{z}}^{\dagger}
$$


which are the Fischer duals of $\underline{z}$ and $\underline{z}^{\dagger}$, and may be seen as refinements of the Euclidean Dirac operator since

$$
\partial=2\left(\partial_{\underline{z}}^{\dagger}-\partial_{\underline{z}}\right)
$$

As a side remark, note that the above operators may also be obtained in another way, making explicit use of the complex structure $J$. Indeed, let

$$
\underline{X} \mid=J(\underline{X})=\sum_{j=1}^{n} J\left(e_{j}\right) x_{j}+J\left(e_{n+j}\right) y_{j}=\sum_{j=1}^{n}\left(e_{j} y_{j}-e_{n+j} x_{j}\right)
$$

then there arises a second, associated (or "twisted") Dirac operator

$$
\partial_{J}=J(\partial)=\sum_{\alpha=1}^{2 n} J\left(e_{\alpha}\right) \partial_{\alpha}=\sum_{j=1}^{n}\left(e_{j} \partial_{y_{j}}-e_{n+j} \partial_{x_{j}}\right)
$$

associated to $\underline{X} \mid$. We then have that

$$
\begin{aligned}
2 \partial_{\underline{z}}^{\dagger}=\frac{1}{2}(\mathbf{1}+i J)[\partial]=\frac{1}{2} \partial+\frac{i}{2} \partial_{J} \\
2 \partial_{\underline{z}}=-\frac{1}{2}(\mathbf{1}-i J)[\partial]=-\frac{1}{2} \partial+\frac{i}{2} \partial_{J}
\end{aligned}
$$

Now consider a smooth function $F$, taking values in the complex Clifford algebra, then it is called Hermitean monogenic (or $\mathrm{h}-$ monogenic for short) if it is a simultaneous null solution of both Euclidean Dirac operators, i.e. if it fulfills the system

$$
\partial[F]=0=\partial_{J}[F]
$$

or equivalently, if it is a simultaneous null solution of both Hermitean Dirac operators, i.e. if it fulfills the system

$$
\partial_{\underline{z}}[F]=0=\partial_{\underline{z}}^{\dagger}[F] \text {. }
$$

We recall that the two Hermitean Dirac operators $\partial_{\underline{z}}$ and $\partial_{\underline{z}}^{\dagger}$ may be generated (as was the case for the Euclidean Dirac operator $\partial$ ) as generalized gradients through projection on the appropriate invariant subspaces, see [1,2], which moreover guarantees the invariance of the considered system under the unitary group action of $\mathrm{SO}_{J}(2 n) \simeq \mathrm{U}(n)$.

For further use, observe that the Hermitean vector variables and Dirac operators are isotropic on account of the properties of the Witt basis elements, i.e.

$$
(\underline{z})^{2}=\left(\underline{z}^{\dagger}\right)^{2}=0 \quad \text { and } \quad\left(\partial_{\underline{z}}\right)^{2}=\left(\partial_{\underline{z}}^{\dagger}\right)^{2}=0
$$


whence the Laplacian $\Delta=-\partial^{2}=-\partial_{J}^{2}$ allows for the decomposition

$$
\Delta=4\left(\partial_{\underline{z}} \partial_{\underline{z}}^{\dagger}+\partial_{\underline{z}}^{\dagger} \partial_{z}\right)
$$

while also

$$
\underline{z} \underline{z}^{\dagger}+\underline{z}^{\dagger} \underline{z}=|\underline{z}|^{2}=\left|\underline{z}^{\dagger}\right|^{2}=|\underline{X}|^{2}=\left.|\underline{X}|\right|^{2}
$$

\section{Harmonic analysis for $\mathrm{SO}(m)$}

We start with a review of results in the simplest possible case of the space $\mathcal{P}\left(\mathbb{R}^{m} ; \mathbb{C}\right)$ of complex valued polynomials defined in Euclidean space $\mathbb{R}^{m}$, considered first as an $\mathrm{SO}(m)$-module. The action of $\mathrm{SO}(m)$ on polynomials in $\mathcal{P}\left(\mathbb{R}^{m} ; \mathbb{C}\right)$ is the regular representation:

$$
[g \cdot f](\underline{X})=f\left(g^{-1} \cdot \underline{X}\right), \quad g \in \mathrm{SO}(m), \quad f \in \mathcal{P}\left(\mathbb{R}^{m} ; \mathbb{C}\right), \quad \underline{X} \in \mathbb{R}^{m}
$$

If we denote by $\mathcal{H}_{k}$ the space of $k$-homogeneous harmonic polynomials of degree $k$, then each of the spaces

$$
r^{2 p} \mathcal{H}_{k}, \quad p \in \mathbb{N}_{0}:=\mathbb{N} \cup\{0\}, \quad k \in \mathbb{N}_{0}
$$

is a subspace of $\mathcal{P}\left(\mathbb{R}^{m} ; \mathbb{C}\right)$ which is invariant under the $\mathrm{SO}(m)$-action and moreover irreducible. In addition, they form the constituents of the decomposition of $\mathcal{P}\left(\mathbb{R}^{m} ; \mathbb{R}\right)$ according to the following standard triangular diagram:

$$
\begin{array}{cccccc}
\mathcal{H}_{0} & & r^{2} \mathcal{H}_{0} & & r^{4} \mathcal{H}_{0} & \cdots \\
& & & & \\
& \mathcal{H}_{1} & & r^{2} \mathcal{H}_{1} & & \cdots \\
& & \mathcal{H}_{2} & & r^{2} \mathcal{H}_{2} & \cdots \\
& & \mathcal{H}_{3} & & \\
& & & \mathcal{H}_{4} & \cdots
\end{array}
$$

This decomposition, also known in Clifford analysis as the Fischer decomposition, is summarized as

$$
\mathcal{P}\left(\mathbb{R}^{m} ; \mathbb{C}\right)=\bigoplus_{k=0}^{\infty} \bigoplus_{p=0}^{\infty} r^{2 p} \mathcal{H}_{k},
$$

Note that the $j$-th column $(j=0,1,2, \ldots)$ in the above scheme provides the splitting of the space $\mathcal{P}_{j}\left(\mathbb{R}^{m} ; \mathbb{C}\right)$ of $j$-homogeneous polynomials. Although this is a well-known fact in harmonic analysis, we will give an explicit proof using elementary concepts from representation theory, in order to clarify the arguments that will be used in the more advanced settings in the next sections. 
The drawback of decomposition (3.1) is that each $\mathrm{SO}(m)$-irreducible invariant subspace $\mathcal{H}_{k}$ appears with an infinite multiplicity since all subspaces on the same row in the above scheme, namely $r^{2 p} \mathcal{H}_{k}$, with varying $p \in \mathbb{N}_{0}$ and fixed $k \in \mathbb{N}_{0}$, are isomorphic as $\mathrm{SO}(m)$-modules. Yet there is an additional, hidden symmetry in the space $\mathcal{P}\left(\mathbb{R}^{m} ; \mathbb{C}\right)$ which can improve the situation in the sense that the infinitely many copies of the irreducible representations $\mathcal{H}_{k}$ for $\mathrm{SO}(m)$ will be grouped into one single irreducible representation for a suitable Lie algebra $\mathfrak{g}$. The couple $(\mathrm{SO}(m), \mathfrak{g})$ is called a Howe dual pair with respect to a bigger Lie algebra in which $\mathfrak{s o}(m)$, i.e. the Lie algebra of $\mathrm{SO}(m)$, and $\mathfrak{g}$ are commutant to each other. The notion of a reductive dual pair of subgroups of a symplectic group was introduced in the late 1970s by Howe in order to establish a duality relation between representations of different classical Lie groups (see $[18,16,15])$. In order to find this Lie algebra $\mathfrak{g}$ we consider the Weyl algebra $\mathcal{W}$ of differential operators in $\left(\partial_{X_{1}}, \ldots, \partial_{X_{m}}\right)$ with polynomial coefficients. Each such operator acts on $\mathcal{P}\left(\mathbb{R}^{m} ; \mathbb{C}\right)$ in a natural way; the space $\mathcal{P}\left(\mathbb{R}^{m} ; \mathbb{C}\right)$ is a module over $\mathcal{W}$. Note that $\mathcal{P}\left(\mathbb{R}^{m} ; \mathbb{C}\right)$ itself is contained in $\mathcal{W}$ as polynomial differential operators of order zero. The Weyl algebra $\mathcal{W}$ bears the natural structure of a Lie algebra by taking the commutator as the Lie bracket.

It is clear that in the above decomposition (3.1) the operators $X:=\frac{1}{2} r^{2}$ and $Y:=-\frac{1}{2} \Delta$ play a special role. Note that they correspond to each other under natural or Fourier duality, also known in Clifford analysis as Fischer duality. Moreover they both belong to $\mathcal{W}$ and are commuting with $\mathrm{SO}(m)$ so that we are prompted to search for the smallest Lie subalgebra of $\mathcal{W}$ generated by $X$ and $Y$. A direct calculation shows that

$$
[X, Y]=\left[\frac{1}{2} r^{2},-\frac{1}{2} \Delta\right]=\mathbb{E}+\frac{m}{2}
$$

where $\mathbb{E}$ denotes the Euler operator. We then put

$$
H:=\mathbb{E}+\frac{m}{2}
$$

As now $[H, X]=2 X$ and $[H, Y]=-2 Y$, we see that $\{H, X, Y\}$ generates a three-dimensional Lie subalgebra of $\mathcal{W}$ which is isomorphic to the Lie algebra $\mathfrak{s l}(2, \mathbb{R})$. Concluding, the desired Howe dual pair is $(\mathrm{SO}(m), \mathfrak{s l}(2, \mathbb{R}))$. The action of $\mathfrak{s l}(2, \mathbb{R})$ results into the following:

$$
\begin{aligned}
X \cdot r^{2 p} \mathcal{H}_{k} & =r^{2 p+2} \mathcal{H}_{k} \\
Y \cdot r^{2 p} \mathcal{H}_{k} & =r^{2 p-2} \mathcal{H}_{k} \\
H \cdot r^{2 p} \mathcal{H}_{k} & =r^{2 p} \mathcal{H}_{k}
\end{aligned}
$$


In the above scheme this corresponds to respectively a shift to the right in each row, a shift to the left in each row and a local trampling, which already indicates that this action will force us to consider each row as one entity.

Now we shall illustrate the main ideas of the Howe dual pair technique in this simplest case, by (re)proving the decomposition (3.1) in an elementary way using representation theory techniques (see [18]). Here and in the subsequent sections we will make use of the following classical result for the Lie algebra $\mathfrak{s l}(2, \mathbb{R})$ (see e.g. [13]).

Lemma 1. If $\{H, X, Y\}$ are the generators of the Lie algebra $\mathfrak{s l}(2, \mathbb{R})$ and $w$ is an eigenvector of $H$ with eigenvalue $\lambda$, which is moreover annihilated by $Y$, then for all $\ell \in \mathbb{N}$

$$
Y X^{\ell} w=\ell(\lambda+\ell-1) X^{\ell-1} w
$$

and hence $Y^{\ell} X^{\ell} w=C_{\lambda, \ell} w$ with $C_{\lambda, \ell}=\ell !(\lambda+\ell-1)(\lambda+\ell-2) \cdots \lambda$.

As a first step in the proof we introduce some new notions. Let $\left\{H_{k}^{(j)}: j \in J_{k}\right\}$ be a basis for the $\mathrm{SO}(m)$-module $\mathcal{H}_{k}$. The set

$$
\left\{H_{k}^{(j)}: k \in \mathbb{N}_{0}, j \in J_{k}\right\}
$$

is a set of so-called singular vectors, which means that each of these polynomials is annihilated by $Y$. Moreover, for all $k \in \mathbb{N}_{0}$ and $j \in J_{k}$, the repeated action of $X$ generates the following $\mathfrak{s l}(2, \mathbb{R})$-module:

$$
\mathbb{V}_{k}^{(j)}:=\operatorname{span}_{\mathbb{C}}\left\{X^{\ell} H_{k}^{(j)}: \ell \in \mathbb{N}_{0}\right\}
$$

Note that the vectors $X^{\ell} H_{k}^{(j)}$ all have different degrees of homogeneity and thus form a basis for $\mathbb{V}_{k}^{(j)}$.

Lemma 2. The $\mathfrak{s l}(2, \mathbb{R})$-modules $\mathbb{V}_{k}^{(j)}$ are all infinite-dimensional and irreducible. The modules $\mathbb{V}_{k}^{(i)}$ and $\mathbb{V}_{p}^{(j)}$ are isomorphic if and only if $k=p$.

Proof. That the modules $\mathbb{V}_{k}^{(j)}$ are irreducible is a consequence of Lemma 1, since the $H$-eigenvalue $\lambda$ of $H_{k}^{(j)}$ equals $k+\frac{m}{2}>0$. Moreover two modules $\mathbb{V}_{k}^{(i)}$ and $\mathbb{V}_{p}^{(j)}$ can only be isomorphic if their generating singular vectors have the same $H$-eigenvalue, i.e. when $k=p$. 
In the next step of our proof we show that for each $k$-homogeneous polynomial $P_{k}(\underline{X})$ there exist unique harmonic polynomials $H_{k-2 \ell}(\underline{X})$ such that

$$
P_{k}(\underline{X})=\sum_{\ell=0}^{\kappa} X^{\ell} H_{k-2 \ell}(\underline{X})
$$

where we have introduced the notation $\kappa=\left\lfloor\frac{k}{2}\right\rfloor$. Indeed, in view of Lemma 1 , there exists a non-trivial constant $c_{\kappa}$ such that

$$
Y^{\kappa}\left(P_{k}-c_{\kappa} X^{\kappa} Y^{\kappa} P_{k}\right)=0
$$

Putting

$$
H_{k-2 \kappa}=c_{\kappa} Y^{\kappa} P_{k} \quad \text { and } \quad P_{k}^{(1)}=P_{k}-X^{\kappa} H_{k-2 \kappa},
$$

it is immediately seen that $H_{k-2 \kappa}$ is harmonic, that $P_{k}^{(1)}$ is in the kernel of $Y^{\kappa}$ and that both are uniquely determined. Repeating this argument proves the statement on $P_{k}(\underline{X})$ and hence also the decomposition (3.1).

In fact each of the spaces of polynomials $\mathbb{V}_{k}^{(j)}$ is a realization of the Verma module with lowest weight $\lambda_{k}=k+\frac{m}{2}$, which is an irreducible $\mathfrak{s l}(2, \mathbb{R})$-module; we denote this Verma module by $\mathbb{I}_{k}$. Similarly the space of $k$-homogeneous harmonic polynomials $\mathcal{H}_{k}$ is a realization of the irreducible $\mathrm{SO}(m)$-module with weight $(k, 0, \ldots, 0)$, which we denote by $\mathbb{H}_{k}$. For each $k \in \mathbb{N}_{0}$, the tensor product $\mathbb{I}_{k} \otimes \mathbb{H}_{k}$ then is an irreducible $\mathfrak{s l}(2, \mathbb{R}) \times \mathfrak{s o}(m, \mathbb{R})$ module, realized by the row $\bigoplus_{p=0}^{\infty} r^{2 p} \mathcal{H}_{k}$ in the triangular diagram. When regarded as an $\mathfrak{s l}(2, \mathbb{R})$-module this tensor product contains as many copies of $\mathbb{I}_{k}$ as the dimension of $\mathbb{H}_{k}$, while when regarded as an $\mathfrak{s o}(m, \mathbb{R})$-module it contains infinitely many copies of $\mathbb{H}_{k}$ since $\mathbb{I}_{k}$ has infinite dimension. The traditional decomposition (3.1) may thus be reformulated (see, e.g., [18, 16]) as follows.

Theorem 1. Under the joint action of $\mathfrak{s l}(2, \mathbb{R}) \times \mathrm{SO}(m)$, the space of complex valued polynomials $\mathcal{P}\left(\mathbb{R}^{m} ; \mathbb{C}\right)$ is isomorphic with the multiplicity free irreducible direct sum

$$
\bigoplus_{k=0}^{\infty} \mathbb{I}_{k} \otimes \mathbb{H}_{k}
$$

where $\mathbb{I}_{k}$ denotes the Verma module with lowest weight $k+\frac{m}{2}$, and $\mathbb{H}_{k}$ denotes the irreducible $\mathrm{SO}(m)$-module with weight $(k, 0, \ldots, 0)$. 


\section{Harmonic analysis for $\mathrm{U}(n)$}

We shall now apply the same methods to the same space $\mathcal{P}\left(\mathbb{R}^{2 n} ; \mathbb{C}\right)$ of complex valued polynomials defined in Euclidean space $\mathbb{R}^{2 n}$, but this time considered as a $\mathrm{U}(n)$-module. This is again an explicit formulation of a special case of the abstract scheme formulated in $[18,19]$. As in the previous section, the final aim is to obtain a multiplicity free irreducible direct sum decomposition of the form

$$
\mathcal{P}\left(\mathbb{R}^{2 n} ; \mathbb{C}\right) \simeq \bigoplus_{a, b} \mathbb{I}_{a, b} \otimes \mathbb{H}_{a, b}
$$

where each $\mathbb{I}_{a, b} \otimes \mathbb{H}_{a, b}$ is a $\mathfrak{u}(n) \times \widetilde{\mathfrak{g}}-$ module, $(\mathrm{U}(n), \widetilde{\mathfrak{g}})$ being a Howe dual pair still to be determined. The action of $\mathrm{U}(n)$ is given by

$$
[u \cdot f](\underline{X})=f\left(u^{-1} \cdot \underline{X}\right), \quad u \in \mathrm{U}(n), \quad f \in \mathcal{P}\left(\mathbb{R}^{2 n} ; \mathbb{C}\right), \quad \underline{X} \in \mathbb{R}^{2 n}
$$

where the group $\mathrm{U}(n)$ is seen as isomorphic with the subgroup $\mathrm{SO}_{J}(2 n)$ consisting of all $\mathrm{SO}(2 n)$ elements commuting with the so-called complex structure $J \in \mathrm{SO}(2 n)$, as introduced in Section 2. In view of the fact that each complex valued polynomial in the variables $\left(x_{1}, \ldots, x_{n}, y_{1}, \ldots, y_{n}\right)$ may also be written as a polynomial in the variables $\left(z_{1}, \ldots, z_{n}, z_{1}^{c}, \ldots, z_{n}^{c}\right)$, i.e.

$$
f(\underline{X})=f\left(x_{1}, \ldots, x_{n}, y_{1}, \ldots, y_{n}\right)=\widetilde{f}\left(z_{1}, \ldots, z_{n}, z_{1}^{c}, \ldots, z_{n}^{c}\right),
$$

the question to be answered, namely which polynomials in $\mathcal{P}\left(\mathbb{R}^{2 n} ; \mathbb{C}\right)$ are invariant under the action of $\mathrm{SO}_{J}(2 n)$, may thus be reformulated as which polynomials $\tilde{f}\left(z_{1}, \ldots, z_{n}, z_{1}^{c}, \ldots, z_{n}^{c}\right)$ are invariant under the action of $\mathrm{U}(n)$. As is well-known the space $\mathcal{I}$ of $\mathrm{U}(n)$-invariant polynomials in $\mathcal{P}\left(\mathbb{R}^{2 n} ; \operatorname{End}(\mathbb{C})\right)$ is the space with basis

$$
\left(1, r^{2}, r^{4}, \ldots, r^{2 p}, \ldots\right)
$$

where the generator $r^{2}$ can be written as:

$$
r^{2}=\sum_{j=1}^{n} x_{j}^{2}+y_{j}^{2}=\sum_{j=1}^{n} z_{j} z_{j}^{c}=\sum_{j=1}^{n}\left|z_{j}\right|^{2} .
$$

With the generator $r^{2}$ there corresponds the Laplace operator

$$
\Delta=\sum_{j=1}^{n} \partial_{x_{j} x_{j}}^{2}+\partial_{y_{j} y_{j}}^{2}=4 \sum_{j=1}^{n} \partial_{z_{j}} \partial_{z_{j}^{c}}
$$


so we are lead to consider the space $\mathcal{H}^{\mathbb{C}}$ of harmonic polynomials in the complex variables $\left(z_{1}, \ldots, z_{n}, z_{1}^{c}, \ldots, z_{n}^{c}\right)$. The subspace $\mathcal{H}_{k}^{\mathbb{C}}$ of complex valued $k$-homogeneous harmonic polynomials may be decomposed as

$$
\mathcal{H}_{k}^{\mathbb{C}}=\bigoplus_{a=0}^{k} \mathcal{H}_{a, k-a}
$$

where $\mathcal{H}_{a, b}$ stands for the space of the complex valued harmonic polynomials which are $a$-homogeneous in the variables $z_{j}$ and at the same time $b$-homogeneous in the variables $z_{j}^{c}$, i.e.

$$
H_{a, b}\left(\lambda z_{1}, \ldots, \lambda z_{n}, \mu z_{1}^{c}, \ldots, \mu z_{n}^{c}\right)=\lambda^{a} \mu^{b} H_{a, b}\left(z_{1}, \ldots, z_{n}, z_{1}^{c}, \ldots, z_{n}^{c}\right) .
$$

This leads to the direct sum decomposition

$$
\mathcal{P}\left(\mathbb{R}^{2 n} ; \mathbb{C}\right)=\bigoplus_{p=0}^{\infty} \bigoplus_{k=0}^{\infty} \bigoplus_{a=0}^{k} r^{2 p} \mathcal{H}_{a, k-a}
$$

where the constituents

$$
r^{2 p} \mathcal{H}_{a, k-a}, \quad p \in \mathbb{N}_{0}, \quad k \in \mathbb{N}_{0}, \quad a=0, \ldots, k
$$

are irreducible invariant subspaces under the action of $\mathrm{U}(n)$. The corresponding pyramidal diagram is depicted in the figure below. Note that the decomposition of the complex valued polynomials of a fixed degree $k$ of homogeneity is obtained by considering in this scheme vertical planes perpendicular to the first bisector in the $(a, b)$-plane, yielding triangles if $k$ is even, and trapezia if $k$ is odd.

The smallest Lie subalgebra of the Weyl algebra $\mathcal{W}^{\mathbb{C}}$ of complex polynomial differential operators, generated by the polynomial $r^{2}$ and its dual operator $\Delta$ is again $\mathfrak{s l}(2, \mathbb{R})$ since

$$
[X, Y]=\left[\frac{1}{2} r^{2},-\frac{1}{2} \Delta\right]=\mathbb{E}+n=H
$$

However there is an additional natural invariant differential operator coming into play. Indeed, it is easily seen that $\mathbb{E}$ decomposes as $\mathbb{E}=\mathbb{E}_{z}+\mathbb{E}_{z^{c}}$, with

$$
\mathbb{E}_{z}=\sum_{j=1}^{n} z_{j} \partial_{z_{j}} \quad \text { and } \quad \mathbb{E}_{z^{c}}=\sum_{j=1}^{n} z_{j}^{c} \partial_{z_{j}^{c}}
$$

being the Euler operators in the complex variables and in their conjugates, respectively. Obviously the latter operators are invariant, and so is their difference $\mathbb{E}_{z^{c}}-\mathbb{E}_{z}$. Moreover as

$$
\left[r^{2}, \mathbb{E}_{z}\right]=\left[r^{2}, \mathbb{E}_{z^{c}}\right]=-r^{2} \quad \text { and } \quad\left[\Delta, \mathbb{E}_{z}\right]=\left[\Delta, \mathbb{E}_{z^{c}}\right]=\Delta
$$


the operator $\mathbb{E}_{z^{c}}-\mathbb{E}_{z}$ commutes with both $X$ and $Y$ and trivially with $H=\mathbb{E}_{z}+\mathbb{E}_{z^{c}}+n$, and so do all operators of the form $\mathbb{E}_{z^{c}}-\mathbb{E}_{z}+c$, where we choose the constant $c=n$ in accordance with standard notations to be encountered in Section 6.

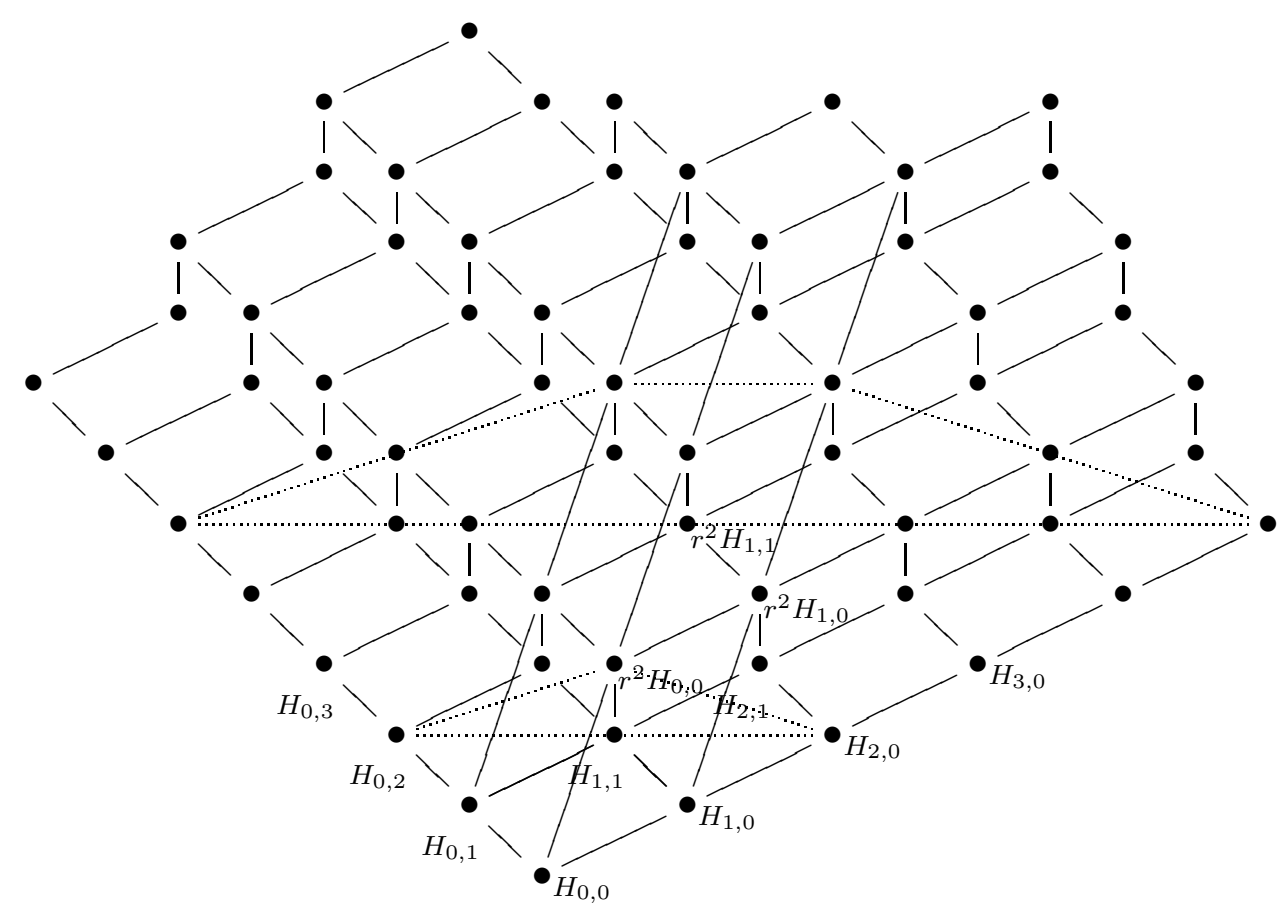

Figure 1: Fischer decomposition of harmonic polynomials w.r.t. $\mathrm{U}(n)$

So it turns out that our Lie subalgebra is reductive, i.e. the direct sum of the three-dimensional Lie algebra generated by $\{H, X, Y\}$, which is isomorphic with $\mathfrak{s l}(2, \mathbb{R})$, and the one-dimensional abelian Lie algebra $\mathfrak{s l}(1, \mathbb{R})$ generated by $\left\{\mathbb{E}_{z^{c}}-\mathbb{E}_{z}+n\right\}$, which is isomorphic with $\mathbb{R}$. In fact the Howe dual pair for complex harmonic polynomials here is $(\mathrm{U}(n), \mathfrak{g l}(2, \mathbb{R}))$, with $\mathfrak{g l}(2, \mathbb{R})$ the real general linear algebra in two dimensions. Irreducible $\mathfrak{g l}(2, \mathbb{R})$-modules will be represented by their weight vector $\underline{\lambda}=\left(\lambda_{1}, \lambda_{2}\right)$, which can be seen as the vector containing the eigenvalues for the Cartan subalgebra $\mathfrak{h} \subset \mathfrak{g l}(2, \mathbb{R})$. Let us then investigate the action of $\mathfrak{g l}(2, \mathbb{R})$ :

- the action of $r^{2}$ joins $r^{2 p} \mathcal{H}_{a, k-a}$ with $r^{2(p+1)} \mathcal{H}_{a, k-a}$, and corresponds in the above scheme with a translation over the vector $(1,1,1)$ in the $(a, b, p)$-space 
- the action of $\Delta$ joins $r^{2 p} \mathcal{H}_{a, k-a}$ with $r^{2(p-1)} \mathcal{H}_{a, k-a}$, and corresponds with the inverse translation over the vector $(-1,-1,-1)$

- the respective actions of $\mathbb{E}_{z}$ and $\mathbb{E}_{z^{c}}$ keep the spaces $r^{2 p} \mathcal{H}_{a, k-a}$ unaltered

Again, we may already predict from these observations that all subspaces along the same space diagonal will have to be considered as one entity as a consequence of the action of $\mathfrak{g l}(2, \mathbb{R})$, and in view of a multiplicity free decomposition.

So let us decompose the $\mathrm{U}(n)$-module $\mathcal{P}\left(\mathbb{R}^{2 n} ; \mathbb{C}\right)$ under the combined action of the dual pair $\mathfrak{g l}(2, \mathbb{R}) \times \mathfrak{u}(n)$. Choosing a basis $\left\{H_{a, b}^{(j)}: j \in J_{a, b}\right\}$ for each irreducible $\mathrm{U}(n)$-module $\mathcal{H}_{a, b}$, with $a, b \in \mathbb{N}_{0}$ fixed, we again get a set of singular vectors, labeled by three parameters $a, b$ and $j$. The repeated action of $X$ then generates the module $\mathbb{V}_{a, b}^{(j)}$ defined by

$$
\mathbb{V}_{a, b}^{(j)}:=\operatorname{span}_{\mathbb{C}}\left\{X^{\ell} H_{a, b}^{(j)}: \ell \in \mathbb{N}_{0}\right\} .
$$

Each of the spaces of polynomials $\mathbb{V}_{a, b}^{(j)}$ is a realization of the Verma module with lowest weight $\lambda=a+b+n$, which is an irreducible $\mathfrak{s l}(2, \mathbb{R})$-module; as in the previous section we denote this Verma module by $\mathbb{I}_{a+b}$ and we put $\mathbb{I}_{a, b}=$ $\mathbb{C}_{b-a+n} \otimes \mathbb{I}_{a+b}$, where $\mathbb{C}_{b-a+n}$ is a representation of $\mathfrak{s l}(1, \mathbb{R})$, which is identified with $\mathbb{R}$. For completeness let us mention that such a representation $\mathbb{C}_{\alpha}$ of $\mathfrak{s l}(1, \mathbb{R})$ is given by

$$
\rho_{\alpha}: \mathbb{R} \rightarrow \operatorname{End}(\mathbb{C}) \simeq \mathbb{C}, x \mapsto \alpha x
$$

and that here $\alpha=b-a+n$ is exactly the eigenvalue of the generator $\mathbb{E}_{z^{c}}-\mathbb{E}_{z}+n$ of $\mathfrak{s l}(1, \mathbb{R})$ acting on homogeneous polynomials. Similarly the space of $(a, b)$-homogeneous harmonic polynomials $\mathcal{H}_{a, b}$ is a realization of the irreducible $\mathrm{U}(n)$-module with weight $(b, 0, \ldots, 0,-a)$, which we denote by $\mathbb{H}_{a, b}$. For all $(a, b)$, the tensor product $\mathbb{I}_{a, b} \otimes \mathbb{H}_{a, b}$ then is an irreducible $\mathfrak{g l}(2, \mathbb{R}) \times \mathfrak{u}(n)$-module, realized by the space diagonal $\bigoplus_{p=0}^{\infty} r^{2 p} \mathcal{H}_{a, b}$ in the pyramidal diagram. When regarded as a $\mathfrak{g l}(2, \mathbb{R})$-module it contains as many copies of $\mathbb{I}_{a, b}$ as the dimension of $\mathbb{H}_{a, b}$, while when regarded as a $\mathfrak{u}(n)$-module it contains infinitely many copies of $\mathbb{H}_{a, b}$ since $\mathbb{I}_{(a, b)}$ has infinite dimension. The obtained decomposition may thus be reformulated as follows.

Theorem 2. Under the joint action of $\mathfrak{g l}(2, \mathbb{R}) \times \mathrm{U}(n)$, the space $\mathcal{P}\left(\mathbb{R}^{2 n} ; \mathbb{C}\right)$ is isomorphic with the multiplicity free irreducible direct sum decomposition

$$
\bigoplus_{a, b=0}^{\infty} \mathbb{I}_{a, b} \otimes \mathbb{H}_{a, b}
$$

where $\mathbb{I}_{a, b}=\mathbb{C}_{b-a+n} \otimes \mathbb{I}_{a+b}, \mathbb{I}_{a+b}$ is the Verma module with lowest weight $a+b$ $+n$ and $\mathbb{H}_{a, b}$ denotes the irreducible $\mathrm{U}(n)$-module with weight $(b, 0, \ldots, 0,-a)$. 


\section{Euclidean Clifford analysis}

We will now show that a similar scheme applies to classical Clifford analysis. To this end we consider the space $\mathcal{P}\left(\mathbb{R}^{m} ; \mathbb{S}\right)$ of polynomials defined in Euclidean space $\mathbb{R}^{m}$ and taking values in an irreducible representation $\mathbb{S}$ of the Clifford algebra $\mathbb{C}_{m} \equiv \mathcal{C} \ell\left(E_{\mathbb{C}},-Q_{\mathbb{C}}\right)$. Such a representation $\mathbb{S}$ is usually called a spinor space and realized inside the Clifford algebra $\mathbb{C}_{m}$ using a suitable primitive idempotent (see Section 6). The final aim is to obtain a multiplicity free irreducible direct sum decomposition of the form

$$
\mathcal{P}\left(\mathbb{R}^{m} ; \mathbb{S}\right) \simeq \bigoplus_{k} \mathbb{I}_{k} \otimes \mathbb{M}_{k}
$$

where each $\mathbb{I}_{k} \otimes \mathbb{M}_{k}$ is a $\operatorname{Pin}(m) \times \widetilde{\mathfrak{g}}$-module, $(\operatorname{Pin}(m), \widetilde{\mathfrak{g}})$ being a Howe dual pair still to be determined.

First we consider the action of $\operatorname{Pin}(m)$ on $\mathcal{P}\left(\mathbb{R}^{m} ; \mathbb{S}\right)$ given by

$[s \cdot f](\underline{X})=\rho(s)\left[f\left(s^{-1} \underline{X} s\right)\right]=s f\left(s^{-1} \underline{X} s\right), f \in \mathcal{P}\left(\mathbb{R}^{m} ; \mathbb{S}\right), s \in \operatorname{Pin}(m), \underline{X} \in \mathbb{R}^{m}$

where $\rho(s)$ denotes the representation of $s \in \operatorname{Pin}(m)$ in $\mathbb{S}$. We shall also need the action of $\operatorname{Pin}(m)$ on the space $\mathcal{P}\left(\mathbb{R}^{m} ; \operatorname{End}(\mathbb{S})\right)$. The space $\operatorname{End}(\mathbb{S})$ is isomorphic (as a vector space) with the Clifford algebra $\mathbb{C}_{m}$ when $m$ is even, or with its even part when $m$ is odd. Let $s \mapsto \widehat{s}$ denote the main involution on the Clifford algebra; it has eigenvalues \pm 1 , the corresponding eigenspaces being the even and odd part of the Clifford algebra. The action of $\operatorname{Pin}(m)$ on $\mathcal{P}\left(\mathbb{R}^{m} ; \operatorname{End}(\mathbb{S})\right)$ is then given by

$$
\left.[s \cdot f](\underline{X})=s f\left(s^{-1} \underline{X} s\right)\right] \widehat{s}^{-1}, f \in \mathcal{P}\left(\mathbb{R}^{m} ; \operatorname{End}(\mathbb{S})\right), s \in \operatorname{Pin}(m), \underline{X} \in \mathbb{R}^{m}
$$

As is well-known we find the space $\mathcal{I}$ of $\operatorname{Pin}(m)$-invariant polynomials in $\mathcal{P}\left(\mathbb{R}^{m} ; \operatorname{End}(\mathbb{S})\right)$ to be the space with basis $\left(1, \underline{X}, \underline{X}^{2}, \underline{X}^{3}, \ldots, \underline{X}^{p}, \ldots\right)$ which may be written as

$$
\operatorname{span}_{\mathbb{C}}\left(1, \underline{X}^{2}, \underline{X}^{4}, \ldots\right) \oplus \operatorname{span}_{\mathbb{C}}\left(\underline{X}, \underline{X}^{3}, \underline{X}^{5}, \ldots\right)
$$

and becomes a unital superalgebra or $\mathbb{Z}_{2}$-graded algebra, reflecting the natural grading of the Clifford algebra given by its decomposition into the even subalgebra and the odd subspace. There is a natural action of elements in $\mathcal{I}$ on $\mathbb{S}$ valued polynomials given by the natural action of elements in $\operatorname{End}(\mathbb{S})$ on $\mathbb{S}$.

The Pin $(m)$-invariant differential operator corresponding, under the natural duality, with the generator $\underline{X}$ of the above graded algebra, is the Dirac operator $\partial$. Its polynomial null solutions are called spherical monogenics; 
we denote by $\mathcal{M}_{k}$ the space of $k$-homogeneous spherical monogenics with values in the spinor space $\mathbb{S}$. Then each of the spaces

$$
\underline{X}^{p} \mathcal{M}_{k}, \quad p \in \mathbb{N}_{0}, \quad k \in \mathbb{N}_{0}
$$

is an invariant subspace of $\mathcal{P}\left(\mathbb{R}^{m} ; \mathbb{S}\right)$ under the action of $\operatorname{Pin}(m)$, which is moreover irreducible, leading to the desired decomposition of $\mathcal{P}\left(\mathbb{R}^{m} ; \mathbb{S}\right)$ into

$$
\mathcal{P}\left(\mathbb{R}^{m} ; \mathbb{S}\right)=\bigoplus_{k=0}^{\infty} \bigoplus_{p=0}^{\infty} \underline{X}^{p} \mathcal{M}_{k}
$$

or more explicitly according to the standard triangular diagram

$$
\begin{aligned}
& \mathcal{M}_{0} \quad \underline{X} \mathcal{M}_{0} \quad \underline{X}^{2} \mathcal{M}_{0} \quad \underline{X}^{3} \mathcal{M}_{0} \quad \cdots \\
& \mathcal{M}_{1} \quad \underline{X}_{\mathcal{M}_{1}} \quad \frac{X^{2}}{X} \mathcal{M}_{1} \quad \cdots \\
& \begin{array}{ccc}
\mathcal{M}_{2} & \underline{X}_{2} & \cdots \\
\mathcal{M}_{3} & \cdots
\end{array}
\end{aligned}
$$

In this diagram each column provides the splitting of the subspace of homogeneous spinor valued polynomials. The easiest way to prove this decomposition, is to show that the tensor product $\mathcal{H}_{k} \otimes \mathbb{S}$ of two irreducible $\operatorname{Pin}(m)$-modules decomposes as

$$
\mathcal{H}_{k} \otimes \mathbb{S}=\mathcal{M}_{k} \oplus \underline{X} \mathcal{M}_{k-1} .
$$

Alternatively, one could use the language of singular vectors and isomorphisms between weight spaces. Again this splitting into irreducible Pin $(m)-$ modules is not multiplicity free, since all subspaces situated on the same row in the triangular diagram ( $\underline{X}^{p} \mathcal{M}_{k}$ with $k$ fixed) are isomorphic as $\operatorname{Pin}(m)-$ modules. As before it will be possible to join these isomorphic pieces into one single irreducible module for the second partner in the Howe dual pair. There is however an important change, since the Howe dual partner of $\operatorname{Pin}(m)$ will have the structure of a Lie superalgebra.

Definition 1. A Lie superalgebra $\mathfrak{g}$ (over $\mathbb{R}$ or $\mathbb{C}$ ) is a $\mathbb{Z}_{2}$-graded vector space, direct sum of two vector spaces

$$
\mathfrak{g}=\mathfrak{g}_{0} \oplus \mathfrak{g}_{1}
$$

equipped with a graded bracket $[[\cdot, \cdot]]$, satisfying

- the $\mathbb{Z}_{2}$-grading:

$$
\left[\left[a_{i}, a_{j}\right]\right] \in \mathfrak{g}_{i+j(\bmod 2)}, \quad a_{i} \in \mathfrak{g}_{i}, a_{j} \in \mathfrak{g}_{j}
$$


- the graded antisymmetry:

$$
\left[\left[a_{i}, a_{j}\right]\right]=-(-1)^{i j}\left[\left[a_{j}, a_{i}\right]\right], \quad a_{i} \in \mathfrak{g}_{i}, a_{j} \in \mathfrak{g}_{j}
$$

- the generalized Jacobi identity

$$
\begin{array}{r}
(-1)^{i k}\left[\left[a_{i},\left[\left[a_{j}, a_{k}\right]\right]\right]\right]+(-1)^{j i}\left[\left[a_{j},\left[\left[a_{k}, a_{i}\right]\right]\right]\right]+(-1)^{k j}\left[\left[a_{k},\left[\left[a_{i}, a_{j}\right]\right]\right]\right]=0 \\
a_{i} \in \mathfrak{g}_{i}, a_{j} \in \mathfrak{g}_{j}, a_{k} \in \mathfrak{g}_{k}
\end{array}
$$

Note that $\mathfrak{g}_{0}$ is itself a Lie algebra, called the even or bosonic part of $\mathfrak{g}$, while $\mathfrak{g}_{1}$, called the odd or fermionic part of $\mathfrak{g}$, is not. An associative superalgebra $\mathcal{A}=\mathcal{A}_{0} \oplus \mathcal{A}_{1}$ (over $\mathbb{R}$ or $\mathbb{C}$ ) acquires the structure of a Lie superalgebra by taking for the graded bracket the so-called Lie superbracket or supercommutator, which is defined as:

$$
\left[\left[a_{i}, a_{j}\right]\right]=a_{i} a_{j}-(-1)^{i j} a_{j} a_{i}, \quad a_{i} \in \mathcal{A}_{i}, a_{j} \in \mathcal{A}_{j}, \quad i, j=0,1
$$

which in most of the cases is nothing but the usual commutator, except for the case where both $i=1$ and $j=1$, when it is the anticommutator.

We will now search for the Howe dual partner of $\operatorname{Pin}(m)$ in the Lie superalgebra

$$
\mathcal{W}_{\mathbb{S}}:=\mathcal{W} \otimes \operatorname{End}(\mathbb{S})
$$

where, as above, $\mathcal{W}$ stands for the Weyl algebra of polynomial differential operators. This superalgebra $\mathcal{W}_{\mathbb{S}}$ inherits its $\mathbb{Z}_{2}$-grading from the natural $\mathbb{Z}_{2}$-grading of the Clifford algebra $\mathbb{C}_{m}$ with respect to the even and odd parts, via the natural map $\mathbb{C}_{m} \mapsto \operatorname{End}(\mathbb{S})$ given by the spinor representation. Its Lie superalgebra structure then is acquired via the above mentioned graded commutator, here given explicitly by

$$
[[\lambda, \mu]]=\lambda \mu-(-1)^{j k} \mu \lambda, \quad \lambda \in \mathbb{C}_{m}^{(j)}, \quad \mu \in \mathbb{C}_{m}^{(k)}, \quad j, k=0,1
$$

where $\mathbb{C}_{m}^{(0)}$ stands for the even subalgebra of $\mathbb{C}_{m}$ and $\mathbb{C}_{m}^{(1)}$ for the odd subspace.

The dual partner of $\operatorname{Pin}(m)$ will thus arise as a Lie superalgebra generated by $\underline{X}$ and $\partial$, inside the Lie superalgebra $\mathcal{W}_{\mathbb{S}}$. As both generators are odd, we need to compute their graded brackets as their anti-commutators:

- $\{\underline{X}, \underline{X}\}=-2 r^{2}$

- $\{\partial, \partial\}=-2 \Delta$

- $\{\partial, \underline{X}\}=-2\left(\mathbb{E}+\frac{m}{2}\right)$ 
Hence the even part of the Lie superalgebra should contain at least $r^{2}, \Delta$ and $\mathbb{E}+\frac{m}{2}$. As their graded brackets, i.e. their commutators are given by

- $\left[r^{2}, \Delta\right]=-4\left(\mathbb{E}+\frac{m}{2}\right)$

- $\left[\mathbb{E}+n, r^{2}\right]=2 r^{2}$

- $[\mathbb{E}+n, \Delta]=-2 \Delta$

the even part $\left\{r^{2}, \Delta, \mathbb{E}+n\right\}$ closes under the graded bracket to a Lie algebra which is isomorphic with $\mathfrak{s l}(2, \mathbb{R})$. Finally we compute the graded brackets (commutators) of the even with the odd elements:

- $\left[\underline{X}, r^{2}\right]=0$

- $[\underline{X}, \Delta]=-2 \partial$

- $[\underline{X}, \mathbb{E}+n]=-\underline{X}$

and

- $\left[\partial, r^{2}\right]=2 \underline{X}$

- $[\partial, \Delta]=0$

- $[\partial, \mathbb{E}+n]=\partial$

Introducing the standard notations for Lie superalgebras given by

$$
H=\frac{1}{2}(\mathbb{E}+n), \quad E^{+}=\frac{1}{2} r^{2}, \quad E^{-}=-\frac{1}{2} \Delta
$$

and

$$
F^{+}=\frac{1}{2 \sqrt{2}} i \underline{X}, \quad F^{-}=\frac{1}{2 \sqrt{2}} i \partial
$$

we may identify the Howe dual partner

$$
\operatorname{span}_{\mathbb{C}}\left(\frac{1}{2}(\mathbb{E}+n), \frac{1}{2} r^{2},-\frac{1}{2} \Delta\right) \bigoplus \operatorname{span}_{\mathbb{C}}\left(\frac{1}{2 \sqrt{2}} i \underline{X}, \frac{1}{2 \sqrt{2}} i \partial\right)
$$

of $\operatorname{Pin}(m)$ with the well-known Lie superalgebra osp $(1 \mid 2)$, with bosonic generators $E^{+}, E^{-}, H$ and fermionic generators $F^{+}, F^{-}$, the non-vanishing commutation relations of which in the Cartan-Weyl basis read

$$
\begin{aligned}
& {\left[H, E^{ \pm}\right]= \pm E^{ \pm} \quad\left[E^{+}, E^{-}\right]=2 H} \\
& {\left[H, F^{ \pm}\right]= \pm \frac{1}{2} F^{ \pm} \quad\left\{F^{+}, F^{-}\right\}=\frac{1}{2} H} \\
& {\left[E^{ \pm}, F^{\mp}\right]=-F^{ \pm} \quad\left\{F^{ \pm}, F^{ \pm}\right\}= \pm \frac{1}{2} E^{ \pm}}
\end{aligned}
$$


The Howe dual pair thus being the couple $(\operatorname{Pin}(m), \mathfrak{o s p}(1 \mid 2))$, one can have a look at the action of the Lie superalgebra $\mathfrak{o s p}(1 \mid 2)$ on the irreducible invariant subspaces $\underline{X}^{p} \mathcal{M}_{k}$ of $\mathcal{P}\left(\mathbb{R}^{m} ; \mathbb{S}\right)$. First of all, introducing an explicit basis $\left\{M_{k}^{(j)}: j \in J_{k}\right\}$ for the irreducible $\operatorname{Pin}(m)$-module $\mathcal{M}_{k}$, it is easily seen that for all $k \in \mathbb{N}_{0}$ and $j \in J_{k}$, both

$$
\mathbb{V}_{k}^{(j)}:=\left\{\underline{X}^{2 \ell} M_{k}^{(j)}: \ell \in \mathbb{N}_{0}\right\} \quad \text { and } \quad \mathbb{W}_{k}^{(j)}:=\left\{\underline{X}^{2 \ell+1} M_{k}^{(j)}: \ell \in \mathbb{N}_{0}\right\}
$$

define irreducible lowest weight Verma modules for the restriction of $\mathfrak{o s p}(1 \mid 2)$ to its even part $\mathfrak{s l}(2, \mathbb{R})$. On the other hand, the action of the odd part of the dual partner consists of mapping the weight spaces $\mathbb{V}_{k}^{(j)}$ and $\mathbb{W}_{k}^{(j)}$ (isomorphically) into each other as stated in the following lemma.

Lemma 3. For all $k \in \mathbb{N}_{0}$ and $j \in J_{k}$ fixed, the operators $F^{+}=\frac{1}{2 \sqrt{2}} i \underline{X}$ and $F^{-}=\frac{1}{2 \sqrt{2}} i \partial$ act as isomorphisms between consecutive weight spaces along the following diagram:

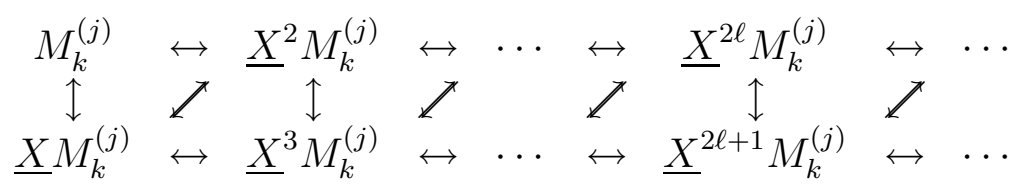

The vertical and diagonal arrows correspond to the action of the operators $F^{+}$and $F^{-}$, whereas the horizontal arrows represent the isomorphisms $E^{+}=\frac{1}{2} r^{2}$ and $E^{-}=-\frac{1}{2} \Delta$ acting between the $\mathfrak{s l}(2, \mathbb{R})$-modules.

Proof. The structure of the $\mathfrak{s l}(2, \mathbb{R})$-modules in the rows is already wellknown. To show that the action of $F^{-}=\frac{1}{2 \sqrt{2}} i \partial$ is nontrivial, it suffices to remark that the one of $\left(F^{-}\right)^{2}=\frac{1}{8} \Delta$ is.

For completeness let us mention that, in order to prove the decomposition announced at the beginning of this section, we can use the same scheme as before based on the action of the $\mathfrak{s l}(2, \mathbb{R})$ generators. The only difference is that, after the action of a suitable power of $F^{-}=\frac{1}{2 \sqrt{2}} i \partial$ on a chosen polynomial, we get a harmonic polynomial, which may then be decomposed into a sum of a monogenic polynomial and a monogenic polynomial multiplied on the left by $\underline{X}$. In fact, the spaces of polynomials $\mathbb{V}_{k}^{(j)}$ and $\mathbb{W}_{k}^{(j)}$ are realizations of the Verma modules with lowest weights $\lambda_{k}=k+\frac{m}{2}$ and $\lambda_{k}=k+1+\frac{m}{2}$ respectively, which are irreducible $\mathfrak{s l}(2, \mathbb{R})$-modules. Their direct sum $\mathbb{V}_{k}^{(j)} \oplus \mathbb{W}_{k}^{(j)}$ then is a realization of an irreducible osp $(1 \mid 2)$-module which we denote by $\widetilde{\mathbb{I}}_{k}$. Similarly the space of $\mathbb{S}$ valued $k$-homogeneous monogenic polynomials $\mathcal{M}_{k}$ is a realization of an irreducible $\operatorname{Pin}(m)$-module which we denote by $\mathbb{M}_{k}$. For each $k \in \mathbb{N}_{0}$ the tensor product $\widetilde{\mathbb{I}}_{k} \otimes \mathbb{M}_{k}$ then 
is an irreducible $\mathfrak{o s p}(1 \mid 2) \times \operatorname{Pin}(m)$-module, which is a realized by the row $\bigoplus_{p=0}^{\infty} r^{2 p} \mathcal{M}_{k}$ in the triangular diagram. When regarded as a osp $(1 \mid 2)-$ module this tensor product contains as many copies of $\widetilde{\mathbb{I}}_{k}$ as the dimension of $\mathbb{M}_{k}$, while when regarded as a $\operatorname{Pin}(m)$-module it contains infinitely many copies of $\mathbb{M}_{k}$ since $\widetilde{\mathbb{I}}_{k}$ has infinite dimension. The Fischer decomposition (5.2) may thus be reformulated as follows.

Theorem 3. Under the joint action of $\mathfrak{o s p}(1 \mid 2) \times \operatorname{Pin}(m)$, the space $\mathcal{P}\left(\mathbb{R}^{m} ; \mathbb{S}\right)$ is isomorphic to the multiplicity free irreducible direct sum

$$
\bigoplus_{k=0}^{\infty} \widetilde{\mathbb{I}}_{k} \otimes \mathbb{M}_{k}
$$

where $\widetilde{\mathbb{I}}_{k}$ is the irreducible $\mathfrak{o s p}(1 \mid 2)$-module defined above and $\mathbb{M}_{k}$ denotes the irreducible $\operatorname{Pin}(m)$-module isomorphic to the space of $\mathbb{S}$ valued, $k$-homogeneous monogenic polynomials.

\section{Hermitean Clifford analysis}

In this section we will make a similar study of the space $\mathcal{P}\left(\mathbb{R}^{2 n} ; \mathbb{S}\right)$ of $\mathbb{S}$ valued polynomials on $\mathbb{R}^{2 n}$ (even dimension). Here we will explicitly realize the spinor space $\mathbb{S}$ in $\mathbb{C}_{2 n}$ as $\mathbb{S}=\mathbb{C}_{2 n} I \simeq \mathbb{C}_{n} I$, where $I$ is the traditional self-adjoint primitive idempotent given by

$$
I=I_{1} \ldots I_{n}
$$

with $I_{j}=\mathfrak{f}_{j} \mathfrak{f}_{j}^{\dagger}=\frac{1}{2}\left(1-i e_{j} e_{n+j}\right), j=1, \ldots, n$. As $\mathfrak{f}_{j} I=0, j=1, \ldots, n$, we also have that $\mathbb{S} \cong \mathbb{C} \Lambda_{n}^{\dagger} I$, where $\mathbb{C} \Lambda_{n}^{\dagger}$ denotes the Grassmann algebra generated by $\left\{\mathfrak{f}_{1}^{\dagger}, \ldots, \mathfrak{f}_{n}^{\dagger}\right\}$. The spinor space $\mathbb{S}$ further decomposes as

$$
\mathbb{S}=\bigoplus_{k=1}^{n} \mathbb{S}^{(k)}=\bigoplus_{k=1}^{n}\left(\mathbb{C} \Lambda_{n}^{\dagger}\right)^{(k)} I
$$

into the so-called homogeneous parts $\mathbb{S}^{(k)}=\left(\mathbb{C} \Lambda_{n}^{\dagger}\right)^{(k)} I, k=1, \ldots, n$, which provide models for fundamental $\mathrm{U}(n)$-representations (see also [1]).

We want to obtain a decomposition of $\mathcal{P}\left(\mathbb{R}^{2 n} ; \mathbb{S}\right)$ into irreducible subspaces under the action of the Lie group $\operatorname{Pin}_{J}(2 n)$, which is the subgroup of Pin $(2 n)$ consisting of those elements which are commuting with $s_{J}=$ $s_{1} s_{2} \ldots s_{n}$, where $s_{j}=\frac{\sqrt{2}}{2}\left(1-e_{j} e_{n+j}\right), j=1, \ldots, n$. The element $s_{J}$ itself belongs to $\operatorname{Spin}(2 n)$ and corresponds to the complex structure $J \in \mathrm{SO}(2 n)$ under the double covering of $\mathrm{SO}(2 n)$ by $\operatorname{Spin}(2 n)$, see Section 2 . In view of this aim, let us briefly summarize the procedure as it has been used in the foregoing cases. 
The approach used in Sections 3 and 4 is based on the scheme described in [16, Section 8]. Suppose that there is an action of a group $G$ on a vector space $E$. The main problem under consideration is to properly understand the decomposition of a suitable space of functions on $E$ under the induced action of the chosen symmetry group $G$. First observe that it is sufficient to consider the corresponding space of polynomials, which do form an algebraic part of the full function space. Hence we are studying the space $\mathcal{P}(E)$ of all polynomials on $E$. In the previous sections we have seen that the resulting decomposition of $\mathcal{P}(E)$ will not be multiplicity free; instead, the multiplicities of the subspaces are usually infinite. In [16] it is explained that in order to obtain a multiplicity free decomposition, we need to consider the Weyl algebra $\mathcal{P} \mathcal{D}(E)$ of all differential operators on $E$ with polynomial coefficients and its subalgebra $\mathcal{P D}(E)^{G}$ of those operators which are invariant under the induced action of $G$, and we have to find a suitable set of generators of this subalgebra $\mathcal{P} \mathcal{D}(E)^{G}$. Moreover, we want to find this set of generators in such a way that they form a basis of a Lie subalgebra $\widetilde{\mathfrak{g}}$ of $\mathcal{P} \mathcal{D}(E)^{G}$. The Lie algebra $\widetilde{\mathfrak{g}}$ then forms the dual partner of $G$, needed in order to ensure a multiplicity free decomposition of the space $\mathcal{P}(E)$. The procedure we have used to find the hidden (or dual) symmetry $\widetilde{\mathfrak{g}}$ then starts with an understanding of the structure of the space $\mathcal{I}$ of invariants of the space $\mathcal{P}(E)$ under the action of $G$. The space $\mathcal{I}$ of such invariants clearly is a unital algebra of which we determine the generators $p_{\alpha}, \alpha \in A, A$ being a suitable index set. Their duals with respect to the Fischer inner product, are differential operators $D_{\alpha}(\alpha \in A)$ with constant coefficients which are invariant with respect to the action of $G$. Next we find the Lie subalgebra in $\mathcal{P D}(E)$ generated by $\left(p_{\alpha}, D_{\alpha}\right), \alpha \in A$. This is then the candidate for $\widetilde{\mathfrak{g}}$. The common kernel $\mathcal{H}$ of the set $D_{\alpha}, \alpha \in A$ may be regarded as an analogue of the space of harmonic functions and we expect that the full space of polynomials $\mathcal{P}(E)$ will be isomorphic to the tensor product of $\mathcal{I}$ and $\mathcal{H}$. This kind of splitting of function spaces is usually called "separation of variables". Moreover, as a $\widetilde{\mathfrak{g}} \times G$-module, we expect $\mathcal{P}(E)$ to be isomorphic to a multiplicity free decomposition of the form

$$
\mathcal{P}(E) \simeq \bigoplus_{\kappa \in K} \mathbb{I}_{\kappa} \otimes \mathbb{H}_{\kappa}
$$

where $K$ is an appropriate subset of the set $\widehat{G}$ of isomorphism classes of irreducible $G$-modules, $\mathbb{H}_{\kappa}$ denoting the corresponding representations, and $\mathbb{I}_{\kappa}$ denoting the Howe dual partner for $\mathbb{H}_{\kappa}$. The Howe duality map $\mathbb{H}_{\kappa} \mapsto \mathbb{I}_{\kappa}$ is expected to be one to one. We then prove all needed facts directly, without any reference to $[19]$ or $[16]$. 
As shown in Section 5, we may extend the described scheme to a more general setting involving, in addition, a super vector space $V=V_{0}+V_{1}$ with an action of $G$. We then consider the (super) vector space $\mathcal{P}(E, V)$ of all polynomials on $E$ with values in $V$ and its decomposition under the natural action of $G$. To extend the former procedure to this more general situation, the Weyl algebra $\mathcal{P} \mathcal{D}(E)$ is replaced here by the (super)algebra $\mathcal{P} \mathcal{D}(E) \otimes \operatorname{End}(V)$ of all differential operators on $E$ with coefficients in $\operatorname{End}(V)$. The same procedure as before is used to find the Howe dual partner $\widetilde{\mathfrak{g}}$, however resulting this time into is a sub-superalgebra of the Lie superalgebra $\mathcal{P} \mathcal{D}(E) \otimes \operatorname{End}(V)$. Going through a similar procedure as in the scalar valued case, we expect that the space $\mathcal{P}(E, V)$ again has a multiplicity free decomposition of the form

$$
\mathcal{P}(E, V) \simeq \bigoplus_{\kappa \in K} \mathbb{I}_{\kappa} \otimes \mathbb{H}_{\kappa}
$$

where again, $K$ is an appropriate subset of the set $\widehat{G}$ and $\mathbb{I}_{\kappa}$ are now irreducible representations of the Lie superalgebra $\widetilde{\mathfrak{g}}$. Hence the fundamental change in this more general situation is that the Howe dual partner $\tilde{\mathfrak{g}}$ is a Lie superalgebra now.

The whole setting leads to the identification of a set of constant coefficient differential operators $D_{\alpha}$ on $\mathcal{P}(E, V)$, which form a canonical set of PDE's induced by the choice of the symmetry studied and thus are natural candidates for further function theoretic research. In this section we shall now realize this programme in the case of spinor valued polynomials and the action of the Lie group $\operatorname{Pin}_{J}(2 n)$ defined above; we will show that in this case the set of natural PDE's exactly coincides with the defining equations for Hermitean Clifford analysis.

The action of $\operatorname{Pin}_{J}(2 n)$ on $\mathcal{P}\left(\mathbb{R}^{2 n} ; \mathbb{S}\right)$ is given by

$$
s \cdot \tilde{f}\left(\underline{z}, \underline{z}^{\dagger}\right)=s \tilde{f}\left(s^{-1} \underline{z} s, s^{-1} \underline{z}^{\dagger} s\right), \quad \tilde{f} \in \mathcal{P}\left(\mathbb{R}^{2 n} ; \mathbb{S}\right), \quad s \in \operatorname{Pin}_{J}(2 n)
$$

whereas its action on $\mathcal{P}\left(\mathbb{R}^{2 n} ; \operatorname{End}(\mathbb{S})\right)=\mathcal{P}\left(\mathbb{R}^{2 n} ; \mathbb{C}_{2 n}\right)$ is given by

$$
s \cdot \tilde{f}\left(\underline{z}, \underline{z}^{\dagger}\right)=s \tilde{f}\left(s^{-1} \underline{z} s, s^{-1} \underline{z}^{\dagger} s\right) \widehat{s}^{-1}
$$

Here, we have used the isotropic Hermitean vector variables $\underline{z}$ and $\underline{z}^{\dagger}$ (see Section 2). The key point is that both $\underline{z}$ and $\underline{z}^{\dagger}$ are $\operatorname{Pin}_{J}(2 n)$-invariant elements in $\mathcal{P}\left(\mathbb{R}^{2 n} ; \operatorname{End}(\mathbb{S})\right)$. In fact it may be proved by invariance theory (see e.g. [15]) that the space $\mathcal{I}$ of all $\operatorname{Pin}_{J}(2 n)$-invariant polynomials is spanned by all possible words in the letters $\underline{z}$ and $\underline{z}^{\dagger}$ :

$$
\mathcal{I}=\operatorname{span}_{\mathbb{C}}\left(1, \underline{z}, \underline{z}^{\dagger}, \underline{z} \underline{z}^{\dagger}, \underline{z}^{\dagger} \underline{z}, \underline{z} \underline{z}^{\dagger} \underline{z}, \underline{z}^{\dagger} \underline{z} \underline{z}^{\dagger}, \underline{z} \underline{z}^{\dagger} \underline{z} \underline{z}^{\dagger}, \underline{z}^{\dagger} \underline{z} \underline{z}^{\dagger} \underline{z}, \cdots\right)
$$


or

$$
\mathcal{I}=\operatorname{span}_{\mathbb{C}}\left(w_{l}^{(i)}\left(\underline{z}, \underline{z}^{\dagger}\right): l=0,1,2, \ldots, i=1,2\right)
$$

where $w_{0}^{(1)}=w_{0}^{(2)}=1$ and

$$
\begin{array}{ll}
w_{2 r}^{(1)}\left(\underline{z}, \underline{z}^{\dagger}\right)=\left(\underline{z} \underline{z}^{\dagger}\right)^{r}=|\underline{z}|^{2 r-2} \underline{z} \underline{z}^{\dagger} & w_{2 r+1}^{(1)}\left(\underline{z}, \underline{z}^{\dagger}\right)=|\underline{z}|^{2 r} \underline{z} \\
w_{2 r}^{(2)}\left(\underline{z}, \underline{z}^{\dagger}\right)=\left(\underline{z} \underline{z}^{r}=|\underline{z}|^{2 r-2} \underline{z}^{\dagger} \underline{z}\right. & w_{2 r+1}^{(2)}\left(\underline{z}, \underline{z}^{\dagger}\right)=|\underline{z}|^{2 r} \underline{z}^{\dagger} .
\end{array}
$$

The space $\mathcal{I}$ of all $\operatorname{Pin}_{J}(2 n)$-invariant polynomials becomes a unital graded superalgebra, its grading being inherited from the $\mathbb{Z}_{2}-$ grading on the Clifford algebra.

As a first step towards the decomposition aimed at we remark that the space of polynomials $\mathcal{P}\left(\mathbb{R}^{2 n} ; \mathbb{S}\right)$ may be split according to the degrees of homogeneity in the variables $\underline{z}$ and $\underline{z}^{\dagger}$ and the homogeneous parts of spinor space:

$$
\mathcal{P}\left(\mathbb{R}^{2 n} ; \mathbb{S}\right)=\bigoplus_{k=0}^{n} \bigoplus_{a, b=0}^{\infty} \mathcal{P}_{a, b, k}\left(\mathbb{R}^{2 n} ; \mathbb{S}\right)
$$

Under the natural duality the generators $\underline{z}$ and $\underline{z}^{\dagger}$ of the superalgebra $\mathcal{I}$ correspond to differential operators which are precisely the Hermitean Dirac operators $\partial_{\underline{z}}$ and $\partial_{\underline{z}}^{\dagger}$. So we have to consider the spaces $\mathcal{M}_{a, b, k}$ of $\mathrm{h}$-monogenic $(a, b)$-homogeneous $\mathbb{S}^{(k)}$ valued polynomials in the variables $\left(z_{1}, \ldots, z_{n}, z_{1}^{c}, \ldots, z_{n}^{c}\right)$, denoted as $\left(\underline{z}, \underline{z}^{\dagger}\right)$. Note that the spaces $\mathcal{M}_{a, 0,0}$ and $\mathcal{M}_{0, b, 0}$ are trivial for $a \neq 0$, respectively $b \neq 0$, and do not need to be taken into account in what follows. Also note that the spaces $\mathcal{M}_{a, b, k}$ provide models for irreducible $\mathfrak{s l}(n, \mathbb{C})$-modules described by a specific Young diagram (see [12]). We now claim that the space of spinor valued polynomials decomposes as follows.

Proposition 1. The space $\mathcal{P}\left(\mathbb{R}^{2 n} ; \mathbb{S}\right)$ of spinor valued polynomials may be split according to the action of $\operatorname{Pin}_{J}(2 n)$ as

$$
\mathcal{P}\left(\mathbb{R}^{2 n} ; \mathbb{S}\right)=\bigoplus_{a, b=0}^{\infty} \bigoplus_{k=0}^{n}\left(\mathcal{M}_{a, b, k} \oplus \bigoplus_{p=1}^{\infty} \bigoplus_{i=1,2} w_{p}^{(i)}\left(\underline{z}, \underline{z}^{\dagger}\right) \mathcal{M}_{a, b, k}\right)
$$

The proof will be deferred to the end of this section.

It is clear that the constituents of the decomposition (6.3) are invariant w.r.t. the action of $\operatorname{Pin}_{J}(2 n)$. Now the construction of a Howe dual partner $\mathfrak{g}$ for $\operatorname{Pin}_{J}(2 n)$ is essential in order to obtain a decomposition into irreducible invariant subspaces which is multiplicity free. Again this will be a Lie superalgebra, realized within the superalgebra $\mathcal{W}_{\mathbb{S}}$. Seeing the 
set $\left\{\underline{z}, \underline{z}^{\dagger} ; \partial_{\underline{z}}, \partial_{\underline{z}}^{\dagger}\right\} \subset \mathcal{W}_{\mathbb{S}}$ as the odd part $\mathfrak{g}_{1}$ of the Lie superalgebra searched for, we will first determine their anti-commutators in order to obtain the even part $\mathfrak{g}_{0}$, and then verify whether the algebra $\mathfrak{g}_{0} \oplus \mathfrak{g}_{1}$ closes under the Lie superbracket. Defining the spin-Euler operator $\beta=\sum_{j=1}^{n} \mathfrak{f}_{j}^{\dagger} \mathfrak{f}_{j}$, which acts as a multiplication operator on the spinor space $\mathbb{S}$ and as the constant $k$ on the spaces $\mathbb{S}^{(k)}$ of homogeneous spinors, the following relations are easily verified:

$$
\begin{aligned}
& \left\{\underline{z}, \partial_{\underline{z}}\right\}=\mathbb{E}_{z}+\beta \quad\left\{\underline{z}, \partial_{\underline{z}}^{\dagger}\right\}=0 \\
& \left\{\underline{z}^{\dagger}, \partial_{\underline{z}}^{\dagger}\right\}=\mathbb{E}_{z^{c}}+n-\beta \quad\left\{\underline{z}^{\dagger}, \partial_{\underline{z}}\right\}=0 \\
& \left\{\underline{z}, \underline{z}^{\dagger}\right\}=r^{2} \quad\left\{\partial_{\underline{z}}, \partial_{\underline{z}}^{\dagger}\right\}=\frac{1}{4} \Delta
\end{aligned}
$$

This means that the even subalgebra $\mathfrak{g}_{0}$ is isomorphic to the Lie algebra $\mathfrak{g l}(2, \mathbb{R})=\mathfrak{s l}(2, \mathbb{R}) \oplus \mathfrak{s l}(1)$, since

$$
\mathfrak{g l}(2, \mathbb{R}) \simeq \operatorname{span}_{\mathbb{R}}\left(H, E^{+}, E^{-}\right) \oplus \mathbb{R}(2 Z)
$$

where we have introduced the standard notations $\left(H, E^{ \pm}, Z\right)$ for the generators of $\mathfrak{g l}(2, \mathbb{R})$ :

$$
\begin{aligned}
H & =\frac{1}{2}\left(\mathbb{E}_{z}+\mathbb{E}_{z^{c}}+n\right) & E^{+} & =\frac{1}{2} r^{2} \\
Z & =\frac{1}{2}\left(n-2 \beta+\mathbb{E}_{z^{c}}-\mathbb{E}_{z}\right) & E^{-} & =-\frac{1}{2} \Delta
\end{aligned}
$$

Next, introducing the standard notations $\left(F^{ \pm}, \bar{F}^{ \pm}\right)$for the generators of the odd part $\mathfrak{g}_{1}$ :

$$
\begin{array}{ll}
F^{+}=\frac{\sqrt{2}}{2} \underline{z} & \bar{F}^{+}=\frac{\sqrt{2}}{2} \underline{z}^{\dagger} \\
F^{-}=\sqrt{2} \partial_{\underline{z}}^{\dagger} & \bar{F}^{-}=-\sqrt{2} \partial_{\underline{z}}
\end{array}
$$

it is clear that in this case the Howe dual pair is given by $\left(\operatorname{Pin}_{J}(2 n), \mathfrak{s l}(1 \mid 2)\right)$. The latter is the Lie superalgebra for which the commutation relations in the Cartan-Weyl basis read:

$$
\begin{aligned}
& {\left[H, E^{ \pm}\right]= \pm E^{ \pm} \quad\left[H, F^{ \pm}\right]= \pm \frac{1}{2} F^{ \pm} \quad\left[H, \bar{F}^{ \pm}\right]= \pm \frac{1}{2} \bar{F}^{ \pm}} \\
& {\left[Z, E^{ \pm}\right]=0 \quad\left[Z, F^{ \pm}\right]=\frac{1}{2} F^{ \pm} \quad\left[Z, \bar{F}^{ \pm}\right]=-\frac{1}{2} \bar{F}^{ \pm}} \\
& {\left[E^{ \pm}, F^{ \pm}\right]=0 \quad\left[E^{ \pm}, F^{\mp}\right]=-F^{ \pm} \quad\left[E^{ \pm}, \bar{F}^{\mp}\right]=\bar{F}^{ \pm}} \\
& {\left[E^{ \pm}, \bar{F}^{ \pm}\right]=0 \quad\left[E^{+}, E^{-}\right]=2 H \quad[Z, H]=0} \\
& \left\{F^{ \pm}, F^{ \pm}\right\}=0 \quad\left\{\bar{F}^{ \pm}, \bar{F}^{ \pm}\right\}=0 \quad\left\{F^{ \pm}, \bar{F}^{ \pm}\right\}=E^{ \pm} \\
& \left\{F^{ \pm}, F^{\mp}\right\}=0 \quad\left\{\bar{F}^{ \pm}, \bar{F}^{\mp}\right\}=0 \quad\left\{F^{ \pm}, \bar{F}^{\mp}\right\}=Z \mp H
\end{aligned}
$$

We now introduce a set of singular vectors giving rise to a family of infinitedimensional $\mathfrak{s l}(1 \mid 2)$-modules. To that end let $\left\{M_{a, b, k}^{(j)}: j \in J_{a, b, k}\right\}$ be a basis 
for $\mathcal{M}_{a, b, k}$, where $(a, b)$ and $k$ are being kept fixed, and consider the infinitedimensional space

$$
\mathbb{V}_{a, b, k}^{(j)}=\operatorname{span}_{\mathbb{C}}\left\{w_{\ell}^{(i)}\left(\underline{z}, \underline{z}^{\dagger}\right) M_{a, b, k}^{(j)}: \ell \in \mathbb{N}_{0}, i=1,2\right\}
$$

generated by the singular vector $M_{a, b, k}^{(j)}$. In order to reveal the structure of this $\mathfrak{s l}(1 \mid 2)$-module, we will first focus our attention on its behaviour as a module for the even subalgebra $\mathfrak{g l}(2, \mathbb{R})$. Just as in the Euclidean case (see Section 5), we find that $\mathbb{V}_{a, b, k}^{(j)}$ splits into a direct sum of irreducible $\mathfrak{g l}(2, \mathbb{R})$-modules. Indeed, the space $\mathbb{V}_{a, b, k}^{(j)}$ has the following structure:

$$
\mathbb{V}_{a, b, k}^{(j)}=\mathbb{V}_{a, b, k}^{(j)+} \oplus \mathbb{V}_{a, b, k}^{(j)-}=\left(\mathcal{M}_{a, b, k}^{(j)} \oplus \mathbb{U}_{a, b, k}^{(j)(1)} \oplus \mathbb{U}_{a, b, k}^{(j)(2)}\right) \oplus\left(\mathbb{W}_{a, b, k}^{(j)(1)} \oplus \mathbb{W}_{a, b, k}^{(j)(2)}\right)
$$

where we have put

$$
\begin{aligned}
& \mathbb{U}_{a, b, k}^{(j)(i)}=\operatorname{span}_{\mathbb{C}}\left\{w_{2 r}^{(i)} M_{a, b, k}^{(j)}: r \in \mathbb{N}\right\}, \quad i=1,2 \\
& \mathbb{W}_{a, b, k}^{(j)(i)}=\operatorname{span}_{\mathbb{C}}\left\{w_{2 r-1}^{(i)} M_{a, b, k}^{(j)}: r \in \mathbb{N}\right\}, \quad i=1,2
\end{aligned}
$$

Lemma 4. For all $a, b \in \mathbb{N}_{0}, 0 \leq k \leq n$ and $j \in J_{a, b, k}$, the spaces $\mathbb{W}_{a, b, k}^{(j)(1)}$ and $\mathbb{W}_{a, b, k}^{(j)(2)}$ are infinite-dimensional irreducible $\mathfrak{g l}(2, \mathbb{R})$-modules, which are isomorphic to

$$
\mathbb{I}_{a+k, b-k}=\mathbb{C}_{n-2 k+b-a} \otimes \mathbb{I}_{a+b} .
$$

On the other hand, it is immediately clear that the even subspace contains the irreducible $\mathfrak{g l}(2, \mathbb{R})$-submodule given by

$$
\widetilde{\mathbb{U}}_{a, b, k}^{(j)(1)}=\operatorname{span}_{\mathbb{C}}\left\{|\underline{z}|^{2 \ell} M_{a, b, k}^{(j)}: \ell \in \mathbb{N}_{0}\right\}
$$

obtained by adding the words of even length. This means that there ought to exist a second summand such that

$$
\mathbb{V}_{a, b, k}^{(j)+}=\widetilde{\mathbb{U}}_{a, b, k}^{(j)(1)} \oplus \tilde{\mathbb{U}}_{a, b, k}^{(j)(2)}
$$

the right hand side containing $\mathfrak{g l}(2, \mathbb{R})$-irreducible summands. As the Lie algebra $\mathfrak{g l}(2, \mathbb{R})$ contains the Laplacian as a negative root vector, it is natural to look for a generator of $\widetilde{\mathbb{U}}_{a, b, k}^{(j)(2)}$ in ker $\Delta$. Taking into account the $\mathrm{h}-$ monogenicity of $M_{a, b ; k}^{(j)}$, it is easily verified (see also [12]) that

$$
\left((a+k) \underline{z} \underline{z}^{\dagger}-(b+n-k) \underline{z}^{\dagger} \underline{z}\right) M_{a, b, k}^{(j)} \in \operatorname{ker} \Delta .
$$


This singular vector thus gives rise to the infinite-dimensional $\mathfrak{g l}(2, \mathbb{R})$ module

$$
\widetilde{\mathbb{U}}_{a, b, k}^{(j)(2)}=\operatorname{span}_{\mathbb{C}}\left\{|\underline{z}|^{2 l}\left((a+k) \underline{z} \underline{z}^{\dagger}-(b+n-k) \underline{z}^{\dagger} \underline{z}\right) M_{a, b, k}^{(j)}: l=0,1,2, \ldots\right\}
$$

Note that the constants appearing in (6.4) may be expressed in terms of the scalar operators $\left\{\underline{z}, \partial_{\underline{z}}\right\}$ and $\left\{\underline{z}^{\dagger}, \partial_{\underline{z}}^{\dagger}\right\}$ as well:

$$
\left(\underline{z} \underline{z}^{\dagger}\left\{\underline{z}, \partial_{\underline{z}}\right\}-\underline{z}^{\dagger} \underline{z}\left\{\underline{z}^{\dagger}, \partial_{\underline{z}}^{\dagger}\right\}\right) M_{a, b, k}^{(j)} \in \operatorname{ker}(\Delta) .
$$

This leads to the following result.

Lemma 5. For all $a, b \in \mathbb{N}_{0}, 0 \leq k \leq n$ and $j \in J_{a, b, k}$, the spaces $\widetilde{\mathbb{U}}_{a, b, k}^{(j)(1)}$ and $\widetilde{\mathbb{U}}_{a, b, k}^{(j)(2)}$ are infinite-dimensional irreducible $\mathfrak{g l}(2, \mathbb{R})$-modules, which are isomorphic to

$$
\mathbb{I}_{a+k, b-k}=\mathbb{C}_{n-2 k+b-a} \otimes \mathbb{I}_{a+b+n} \quad \text { and } \mathbb{I}_{a+k+1, b-k+1}=\mathbb{C}_{n-2 k+b-a} \otimes \mathbb{I}_{a+b+n+2},
$$

respectively.

Summarizing we have decomposed each module $\mathbb{V}_{a, b, k}^{(j)}$ into a direct sum of four isomorphic irreducible $\mathfrak{g l}(2, \mathbb{R})$-submodules:

$$
\mathbb{V}_{a, b, k}^{(j)}=\left(\widetilde{\mathbb{U}}_{a, b, k}^{(j)(1)} \oplus \widetilde{\mathbb{U}}_{a, b, k}^{(j)(2)}\right) \oplus\left(\mathbb{W}_{a, b, k}^{(j)(1)} \oplus \mathbb{W}_{a, b ; k}^{(j)(2)}\right)
$$

Note however that the explicit definition for the second summand depends on the constants $a, b$ and $k$, as opposed to the other summands.

Proposition 2. For all $(a, b, k)$ fixed and $j \in J_{a, b, k}$, the module $\mathbb{V}_{a, b, k}^{(j)}$ is an infinite-dimensional irreducible $\mathfrak{s l}(1 \mid 2)$-module.

Proof. It is sufficient to realize that an arbitrary element of $\mathbb{V}_{a, b, k}^{(j)}$ may always be written as a sum containing powers of $|\underline{z}|^{2}$ with any of the following four singular vectors:

$$
\begin{aligned}
M_{a, b, k}^{(j)} & \rightarrow \operatorname{ker}\left(\partial_{\underline{z}}\right) \cap \operatorname{ker}\left(\partial_{\underline{z}}^{\dagger}\right) \subset \operatorname{ker}(\Delta) \\
\underline{z} M_{a, b, k}^{(j)} & \rightarrow \operatorname{ker}\left(\partial_{\underline{z}}^{\dagger}\right) \backslash \operatorname{ker}\left(\partial_{\underline{z}}\right) \subset \operatorname{ker}(\Delta) \\
\underline{z}^{\dagger} M_{a, b, k}^{(j)} & \rightarrow \operatorname{ker}\left(\partial_{\underline{z}}\right) \backslash \operatorname{ker}\left(\partial_{\underline{z}}^{\dagger}\right) \subset \operatorname{ker}(\Delta) \\
\left(c_{1} \underline{z}^{\dagger}-c_{2} \underline{z}^{\dagger} \underline{z}\right) M_{a, b, k}^{(j)} & \rightarrow \operatorname{ker}(\Delta) \backslash\left(\operatorname{ker}\left(\partial_{\underline{z}}\right) \cup \operatorname{ker}\left(\partial_{\underline{z}}^{\dagger}\right)\right)
\end{aligned}
$$

where we have put $c_{1}=a+k$ and $c_{2}=b+n-k$. Let $\ell$ be the biggest exponent of $|\underline{z}|^{2}$ amongst these terms, and consider the action of $\Delta^{\ell}$. In view of Lemma 4 and Lemma 5, this will produce a non-trivial linear combination 
of the singular vectors. Depending on which of these singular vectors will survive, the action of $\partial_{\underline{z}} \partial_{\underline{z}}^{\dagger}$ or $\partial_{\underline{z}}$ or $\partial_{\underline{z}}^{\dagger}$ or none at all, will yield a non-trivial multiple of $M_{a, b, k}^{(j)}$. The only non-trivial case follows from the fact that

$$
\partial_{\underline{z}} \partial_{\underline{z}}^{\dagger}\left(c_{1} \underline{z z}^{\dagger}-c_{2} \underline{z}^{\dagger} \underline{z}\right) M_{a, b, k}^{(j)}=-c_{1} c_{2}\left(1+c_{1}+c_{2}\right) M_{a, b, k}^{(j)} .
$$

Repeated action of $\underline{z}$ and $\underline{z}^{\dagger}$ then generates the whole module, and together with the obvious invariance this proves the statement.

Finally, we have come to the proof of Proposition 1.

Proof of Proposition 1. Let $P_{a, b, k}\left(\underline{z}, \underline{z}^{\dagger}\right)$ be an arbitrary $\mathbb{S}^{(k)}$ valued $(a, b)$ homogeneous polynomial. From the Fischer decomposition for scalar valued polynomials in Section 4, the existence of unique harmonic polynomials $H_{a-l, b-l, k}$ such that

$$
P_{a, b, k}\left(\underline{z}, \underline{z}^{\dagger}\right)=\sum_{l=0}^{\min (a, b)} r^{2 l} H_{a-l, b-l, k}
$$

follows. We now claim that there exist unique $\mathrm{h}-$ monogenic polynomials such that

$H_{a-l, b-l, k}=M_{a-l, b-l, k}+\left\{\begin{array}{l}\underline{z} M_{a-l-1, b-l, k+1} \\ \underline{z}^{\dagger} M_{a-l, b-l-1, k-1}\end{array}\right\}+\left(c_{1} \underline{z} z^{\dagger}-c_{2} \underline{z}^{\dagger} \underline{z}\right) M_{a-l-1, b-l-1, k}$

with $c_{1}=a-l-1+k$ and $c_{2}=b-l-1+n-k$. Once the existence of these $\mathrm{h}$-monogenic polynomials is shown, the proposition follows by an induction argument. So the proof is now reduced to projecting onto weight spaces for irreducible $\mathfrak{s l}(1 \mid 2)$-modules. In view of the characterization of the singular vectors (see (6.6)), it is clear that one starts with the last summand. Putting

$$
M_{a-l-1, b-l-1, k}=-\frac{1}{c_{1} c_{2}\left(1+c_{1}+c_{2}\right)} \partial_{\underline{z}} \partial_{\underline{z}}^{\dagger} H_{a-l, b-l, k}
$$

it is clear that this is indeed an h-monogenic polynomial. We are thus left with the projection

$$
\pi\left(H_{a-l, b-l, k}\right):=\left(1+\frac{c_{1} \underline{z}^{\dagger}-c_{2} \underline{z}^{\dagger} \underline{z}}{c_{1} c_{2}\left(1+c_{1}+c_{2}\right)} \partial_{\underline{z}} \partial_{\underline{z}}^{\dagger}\right) H_{a-l, b-l, k} .
$$

If we then put

$$
\begin{aligned}
& M_{a-l-1, b-l, k+1}=\frac{1}{c_{1}} \partial_{\underline{z}} \pi\left(H_{a-l, b-l, k}\right) \\
& M_{a-l, b-l-1, k-1}=\frac{1}{c_{2}} \partial_{\underline{z}}^{\dagger} \pi\left(H_{a-l, b-l, k}\right)
\end{aligned}
$$


it is readily verified that these polynomials are indeed $\mathrm{h}-$ monogenic. Finally, the last piece is thus given by

$$
M_{a-l, b-l, k}=\left(1-\frac{1}{c_{1}} \underline{z} \partial_{\underline{z}}-\frac{1}{c_{2}} \underline{z}^{\dagger} \partial_{\underline{z}}^{\dagger}\right) \pi\left(H_{a-l, b-l, k}\right)
$$

the $\mathrm{h}-$ monogenicity of which is easily verified.

This result can be reformulated as follows.

Theorem 4. Under the joint action of $\mathfrak{s l}(1 \mid 2) \times \operatorname{Pin}_{J}(2 n)$, the space $\mathcal{P}\left(\mathbb{R}^{2 n} ; \mathbb{S}\right)$ is isomorphic to the multiplicity free irreducible direct sum

$$
\bigoplus_{a, b=0}^{\infty} \bigoplus_{k=0}^{n} \mathbb{I}_{a, b, k} \otimes \mathbb{M}_{a, b, k}
$$

where $\mathbb{I}_{a, b, k}$ denotes the $\mathfrak{s l}(1 \mid 2)$ irreducible module isomorphic to $\mathbb{V}_{a, b, k}^{(j)}$ and where $\mathbb{M}_{a, b, k}$ denotes the irreducible $\operatorname{Pin}_{J}(2 n)$-module isomorphic to the space $\mathcal{M}_{a, b, k}$ of $\mathbb{S}^{(k)}$ valued $(a, b)$-homogeneous $h-m o n o g e n i c$ polynomials.

Acknowledgements. This research is part of a joint project carried out by the Clifford Research Group of Ghent University and the Mathematical Institute of Charles University Prague, supported by the Bilateral Scientific Co-operation between Flanders and Czech Republic. D. Eelbode expresses his sincere gratitude to Prof. V. Souček for his kind hospitality during the academic year 2006-2007, during which this paper was conceived. Moreover, inspiring discussions with Prof. R. Howe during his stay at Prague are gratefully acknowledged as well.

\section{References}

[1] Brackx, F., Bureš, J., De Schepper, H., Eelbode, D., Sommen, F. AND SoučEK, V.: Fundaments of Hermitean Clifford analysis. I. Complex structure. Compl. Anal. Oper. Theory 1 (2007), no. 3, 341-365.

[2] Brackx, F., Bureš, J., De Schepper, H., Eelbode, D., Sommen, F. AND SoučEK, V.: Fundaments of Hermitean Clifford analysis. II. Splitting of $h$-monogenic equations. Complex Var. Elliptic Equ. 52 (2007), no. 10-11, 1063-1079.

[3] Brackx, F., Delanghe, R. and Sommen, F.: Clifford analysis. Research Notes in Mathematics 76. Pitman, Boston, MA, 1982.

[4] Brackx, F., De Knock, B., De Schepper, H., and Sommen, F.: On Cauchy and Martinelli-Bochner integral formulae in Hermitean Clifford analysis. Bull. Braz. Math. Soc. (N.S.) 40 (2009), no. 3, 395-416. 
[5] Brackx, F., De Knock, B., De Schepper, H.: A matrix Hilbert transform in Hermitean Clifford analysis. J. Math. Anal. Appl. 344 (2008), no. 2, 1068-1078.

[6] Brackx, F., De Schepper, H., De Schepper, N. and Sommen, F.: Hermitean Clifford-Hermite polynomials. Adv. Appl. Clifford Algebr. 17 (2007), no. 3, 311-330.

[7] Brackx, F., De Schepper, H. and Sommen, F.: The Hermitian Clifford analysis toolbox. Adv. Appl. Clifford Algebr. 18 (2008), no. 3-4, 451-487.

[8] Brackx, F., De Schepper, H. and Sommen, F.: A theoretical framework for wavelet analysis in a Hermitean Clifford setting. Commun. Pure Appl. Anal. 6 (2007), no. 3, 549-567.

[9] Colombo, F., Sabadini, I., Sommen, F. and Struppa, D. C.: Analysis of Dirac systems and computational algebra. Progress in Mathematical Physics 39. Birkhäuser Boston, Boston, MA, 2004.

[10] Delanghe, R., Sommen, F. And Souček, V.: Clifford algebra and spinor-valued functions, a function theory for the Dirac operator. Kluwer Academic Publishers, Dordrecht, 1992.

[11] Eelbode, D.: Stirling numbers and spin-Euler polynomials. Experiment. Math. 16 (2007), no. 1, 55-66.

[12] Eelbode, D.: Irreducible $\mathfrak{s}(m)$-modules of Hermitean monogenics. Complex Var. Elliptic Equ. 53 (2008), no. 10, 975-987.

[13] Fulton, W. and Harris, J.: Representation theory. A first course, 3rd edition. Springer Verlag, New York, 1996.

[14] Gilbert, J. And Murray, M.: Clifford algebra and Dirac operators in Harmonic analysis. Cambridge Studies in Advanced Mathematics 26. Cambridge University Press, Cambridge, 1991.

[15] Goodman, R. and Wallach, N. R.: Representations and invariants of the classical groups. Encyclopedia of Mathematics and its Applications 68. Cambridge University Press, Cambridge, 1998.

[16] Goodman, R.: Multiplicity-free spaces and the Schur-Weyl-Howe duality. In Representations of real and p-adic Groups, 305-415. Lect. Notes Ser. Inst. Math. Sci. Natl. Univ. Singap. 2. World Scientific, Singapore, 2004.

[17] Gürlebeck, K. And Sprössig, W.: Quaternionic and Clifford calculus for physicists and engineers. J. Wiley \& Sons, Chichester, 1997.

[18] Howe, R.: Transcending classical invariant theory. J. Amer. Math. Soc. 2 (1989), no. 3, 535-552.

[19] Howe, R.: Remarks on classical invariant theory. Trans. Amer. Math. Soc. 313 (1989), no. 2, 539-570.

[20] Howe, R.: Dual pairs in physics: harmonic oscillators, photons, electrons, and singletons. In Applications of groups theory in physics and mahtematical physics (Chicago, 1982), 179-207. Lectures in Appl. Math. 21. Amer. Math. Soc., Providence, RI, 1985. 
[21] Rocha-Chavez, R., Shapiro, M. and Sommen, F.: Integral theorems for functions and differential forms in $\mathbb{C}_{m}$. Chapman \& Hall/CRC Research Notes in Mathematics 428. Chapman\&Hall / CRC, Boca Raton, FL, 2002.

[22] Sabadini, I. and Sommen, F.: Hermitian Clifford analysis and resolutions. Math. Methods Appl. Sci. 25 (2002), no. 16-18, 1395-1413.

[23] Sommen, F. and Peña Peña, D.: A Martinelli-Bochner formula for the Hermitian Dirac equation. Math. Methods Appl. Sci. 30 (2007), no. 9, 1049-1055.

Recibido: 5 de febrero de 2008

Fred Brackx

Clifford Research Group

Faculty of Engineering, Ghent University Galglaan 2, B-9000 Gent, Belgium fbocage. ugent.be

Hennie De Schepper Clifford Research Group

Faculty of Engineering, Ghent University

Galglaan 2, B-9000 Gent, Belgium hds@cage. ugent. be

David Eelbode

Department of Mathematics and Informatics Antwerp University Middelheimlaan 1, B-2020 Antwerpen, Belgium david.eelbode@ua.ac.be

Vladimir Souček Mathematical Institute Faculty of Mathematics and Physics, Charles University Sokolovská 83, 18675 Praha, Czech Republic soucek@karlin.mff.cuni.cz

V. Souček acknowledges support by the institutional grant MSM 0021620839 and by grant GA CR 201/05/2117. F. Brackx and H. De Schepper acknowledge support by the institutional grant BOF06/BIL/012. D. Eelbode acknowledges support by the Mobility Grant of FWO-Vlaanderen. 